\title{
Vibrational Spectroscopy and Chemometrics in Forensic Chemistry: Critical Review, Current Trends and Challenges
}

\author{
Carolina S. Silva, ${ }^{a}$ André Braz ${ }^{b}$ and Maria Fernanda Pimentel ${ }^{\circledR} * a$ \\ ${ }^{a}$ Departamento de Engenharia Química, Universidade Federal de Pernambuco, \\ Av. Prof. Moraes Rego, 1235, Cidade Universitária, 50670-901 Recife-PE, Brazil \\ ${ }^{b}$ Departamento de Química Fundamental, Universidade Federal de Pernambuco, \\ Av. Prof. Moraes Rego, 1235, Cidade Universitária, 50670-901 Recife-PE, Brazil
}

\begin{abstract}
The present manuscript makes an extensive review of the scientific approaches developed in the last decade involving infrared and Raman spectroscopy combined with chemometrics for solving several issues in the investigation of the most relevant forensic traces, such as questioned documents and currency, explosives, gunshot residues, illicit drugs and body fluids. In addition, current trends, main challenges and the adequate use of several chemometric techniques are discussed. Principal component analysis (PCA) was found to be the most used technique. This unsupervised approach, however, has sometimes been misunderstood as a classification technique. Discriminant analysis techniques are widely employed, leaving a range of possibilities for application of class-modeling techniques, particularly in cases of problems regarding only one target class. In addition, increasingly complex dataset structures frequently require nonlinear approaches or flexible techniques such as multivariate curve resolution-alternating least squares (MCR-ALS). Results reporting, however, still lack reliable quality parameters and sample representativeness, posing a significant challenge to the solution of forensic problems. Regarding the analytical techniques, Raman has been playing an important role, especially in the area of questioned documents and of body fluids. Portable and hyperspectral imaging infrared spectrometers have also been showing significant potential in forensic applications.
\end{abstract}

Keywords: forensic, chemometric, Raman spectroscopy, infrared spectroscopy

\section{Introduction}

Forensic chemistry is the core field of forensic science, using and developing chemical methods and techniques for the analysis of materials that are relevant in criminal investigations and legal disputes. ${ }^{1}$ This field presents many challenges from an analytical chemistry point of view. For instance, the array of materials found at crime scenes is diverse, complex and most of the time of an unknown nature. In addition, the quantity of evidence may be insufficient for multiple analysis, yet maintaining the integrity of the material is a legal requirement for counterproof analysis.

Vibrational spectroscopic techniques such as infrared and Raman have gained particular importance in forensic contexts because they combine a number of advantages. ${ }^{2,3}$ They can characterize the structure of a great number

*e-mail: mfp@ufpe.br of organic and inorganic materials found at crime scenes and can help determine their identity. They are nondestructive and relatively simple to use. Moreover, the instrumental versatility of handheld, portable, imaging and sensing approaches allow for a variety of useful analytical possibilities, particularly in loco. ${ }^{4-7}$ Another vibrational technique with forensic application is terahertz spectroscopy (for further reading refer to Burnett et al.). ${ }^{8}$ Its use in forensic laboratories is far less common than Raman and infrared spectroscopies, which is the main reason why it has not been considered within the scope of the present manuscript. Nevertheless, it is expected that the potential of this technique will emerge in future studies.

Muro et al. ${ }^{9}$ have reviewed the application of Raman and infrared spectroscopy to the analysis of trace evidence, ink analysis, biological samples, anthropology, gunshot residues, and illicit drugs, among others. They provided an overview of the scientific approaches developed in recent years and introduced some concepts of multivariate data analysis 
(a.k.a. chemometrics) for more effective analysis of complex spectroscopic data. Since then, several other reviews have been published in forensic chemistry with some focus in chemometrics. For example, Martín-Alberca et al. ${ }^{10}$ reviewed the application of different analytical techniques for analysis of fire debris; Zadora and Menżyk ${ }^{11}$ discussed the potential of spectroscopy (ultraviolet (UV), fluorescence, infrared, Raman) for estimation of time of deposition of bloodstains; Khandasammy et al. ${ }^{12}$ described the advances of Raman spectroscopy for blood, paint and drug analyses; Mazivila and Olivieri ${ }^{13}$ discussed the applications of vibrational spectroscopy and imaging for counterfeit medicines; Cailletaud et al. ${ }^{14}$ and Yu et al..$^{15}$ reviewed the surfaceenhanced Raman spectroscopy (SERS) applications for pharmaceutical and drugs; Kumar and Sharma ${ }^{16,17}$ published two reviews on analytical methodologies employing chemometrics and forensic traces. Despite the important contributions discussed, the authors only provided brief discussions on chemometrics and did not address specific issues encountered at the intersection between vibrational spectroscopy, chemometrics and forensic chemistry. These raise many issues that should be discussed, such as the proper method to employ classification techniques, the organization and division of the training and test sets, validation and substrate influence. The literature clearly shows that there is a wide gap between forensic chemists and chemometricians. On one hand, most forensic chemists are not sufficiently prepared in chemometrics and on the other hand, chemometricians are not familiar with forensic questions.

This manuscript provides a critical discussion of current research studies employing vibrational spectroscopy techniques associated with chemometrics in forensic chemistry contexts, particularly the analysis of the most important types of forensic evidence such as questioned documents and currency, gunshot residues, explosives, illicit drugs and body fluids. We focus the discussion on the main challenges posed by these analyses (for example, mixtures and substrate interferences) and the correct application of the chemometric techniques. Understanding that overlooking the limitations of some methodologies can, on occasion, make them seem limited to a single forensic problem and to the analysis of only a certain type of evidences, this review aims to present the symbiotic relation between analytical chemistry, sampling and statistical representativeness in forensic chemistry.

\section{Chemometrics}

Chemometrics appears as a natural consequence of the qualitative and quantitative extraction of relevant information from spectroscopic data. In many cases, chemometrics provides the statistical basis and confidence levels for reliable identification of compounds of interest, and consequently, helps the experts during the decisionmaking process and collection of relevant evidence for further analysis. A number of chemometric techniques can be employed in forensic analysis, depending on the problem at hand and what information is necessary. Pattern recognition is particularly interesting for forensic purposes because it identifies consistencies in the dataset and can, for instance, classify unknown samples in different categories. ${ }^{18}$ There are different techniques for pattern recognition in forensic chemistry, which can be roughly divided into unsupervised, regression, classification and resolution techniques. Here, we address the main techniques employed in forensic applications, more specifically, a brief consideration of the mathematical theory behind them as well as their advantages and drawbacks.

Before any analysis of data, it is extremely important to apply preprocessing techniques to discard irrelevant information that may be generated by instrumental variation, scattering, spurious radiation or even irrelevant variations in the samples. Data from spectroscopic techniques such as infrared and Raman, usually require some correction, but the application of preprocessing techniques is out of the scope of the present manuscript (for further reading, see Rinnan et al. ${ }^{19}$ Bocklitz et al. ${ }^{20}$ and Lee et al. ${ }^{21}$ works). Nevertheless, it is important to emphasize that preprocessing tools are no miracle-workers and do not replace the acquisition of quality spectra, so fewer modifications of the dataset are preferred. Parsimonious approaches are always a good option in data treatment.

\subsection{Unsupervised techniques}

Unsupervised techniques are extremely useful in forensic chemical analysis, particularly when the expert has no idea of the source of the trace. This is because no prior information is required to build models or make interpretations. Usually, the purpose of using unsupervised techniques is to explore possible clusters and obtain a preliminary discrimination of the samples. As will be shown in this manuscript, the majority of studies employed at least one unsupervised technique before more advanced data treatments. In fact, this practice helps to understand a bit more about the dataset and can solve simple problems.

Principal component analysis (PCA) is the most widely used unsupervised technique, used particularly to extract the relevant information of a dataset in a reduced space, based on the variance of data. It is important to emphasize that PCA is an exploratory technique and not a classification 
technique per se. Despite this, it can assist classification techniques in establishing the class of an unknown sample with a given confidence level. Different classification techniques are addressed at "2.3. Supervised classification" sub-section.

The mathematical aim of PCA is to decompose the $\mathbf{X}$ matrix (containing the observed data) into scores (T) and loadings (P). A PCA model builds a space of new orthogonal variables (principal components or PCs) through a linear combination of the original ones by maximizing the variance in the data. Each PC will retain only a fraction of data variance, ${ }^{22,23}$ which will decrease with the number of components. The plot of scores, which represent samples' coordinates on the new space of variables, provides information about similarities and differences between/ among the samples. The loadings plot corresponds to the weights of the original variables and their contribution to the variability of a specific PC. Thus, the loadings identify the spectral regions related to the variability described in each PC.

PCA is often employed to explore clusters in the dataset but without being able to assign samples to groups according to their similarities. For this, a set of unsupervised classification techniques can be employed, called clustering techniques. ${ }^{24}$ There are two clustering methods, hierarchical and partitioning, which differ in output and algorithm. Hierarchical methods provide multilevel nested results while partitioning offers a single level result. Other clustering techniques can be found in the literature ${ }^{25}$ but only the main ones, hierarchical cluster analysis (HCA) and $\mathrm{K}$-means, will be considered in this manuscript.

HCA is particularly useful when the number of clusters is unknown. There are two approaches for this technique, the agglomerative and the divisive. In the agglomerative method, each sample constitutes a different cluster and an agglomeration process runs until all samples are assembled to a single cluster. The contrary occurs in the divisive method, where the initial cluster contains all samples and a segregation process runs until each sample forms an isolated cluster. ${ }^{25}$ Then, similarities between the samples are calculated, for example, using correlation coefficients, Euclidean, Manhattan or Mahalanobis distances ${ }^{26}$ Afterwards, a linkage method can be defined for the agglomerative approach or a mining method for the divisive approach.

$\mathrm{K}$-means is a clustering technique based on the partitioning approach. In this case, the number of clusters must be known in advance and the initial clusters are randomly assigned. During an iteration, the distance between each individual sample and the clusters' centroid is calculated and the sample is reassigned to the group of the nearest centroid. In every iteration, the centroid is recalculated and this process continues until an ending criterion is met, for example, when there are no more changes in the clusters. ${ }^{25,27}$

As previously mentioned, the identification of clusters often provides important information to forensic scenarios because the similarities and differences between suspicious samples can be identified more easily. Although PCA, HCA and K-means are usually employed for this matter, variance and distances are not always the best approach to find meaningful projections within the dataset. Projection pursuit $(\mathrm{PP})^{28}$ is another unsupervised alternative. The main idea of PP is to represent the dataset in a low dimensional space revealing "interesting structures". To do this, a projection index must be optimized using different functions, which will depend upon the final purpose of the analysis. ${ }^{29-32}$ The kurtosis parameter is one useful projection index for cluster identification (or outlier detection). ${ }^{32-35}$ By minimizing kurtosis, the bimodality of a distribution is maximized, leading to clustered structures. ${ }^{32}$ Ultimately, interpretation of PP results is similar to PCA.

Independent component analysis (ICA) is another unsupervised technique that can be useful for data decomposition. ${ }^{36,37}$ It is based on the assumption that the $\mathbf{X}$ matrix, containing the observed data, is a result of a linear combination of independent sources. The goal is to perform a decomposition in which the statistical independence of the components is maximized. ${ }^{38}$ Different algorithms are employed for this, including FastICA, joint approximate eigenmatrices (JADE), mean-field ICA (MF-ICA), among others. $^{39}$

\subsection{Calibration}

Calibration techniques (also referred to as regression techniques) are supervised tools, in which previous information about the parameter must be provided in order to establish a relationship with the spectral information and find the regression vector; those techniques are useful for quantitative analysis. Principal component regression (PCR) is a calibration technique based on PCA that relates the scores information of the relevant PCs to a parameter of interest, $\mathbf{y}$. The scores relate to $\mathbf{y}$ through a rotation or regression vector, which is used to estimate the parameter for new samples. It is important to observe that, since only the relevant PCs are employed in regression, PCR is able to retain only the essential information to build the regression vector $\mathbf{b}$, which is an advantage when compared to methods like multiple linear regression. ${ }^{26,40,41}$

Partial least squares (PLS or PLSR) ${ }^{42,43}$ is a widely applied regression technique that builds models by 
maximizing the covariance between the spectral information and the property of interest, $\mathbf{y}$. When only one property of interest is being analyzed, $\mathbf{y}$ is a vector and the model is referred to as PLS1; however, when more than one property is being analyzed, $\mathbf{y}$ is a matrix $(\mathbf{Y})$ and the model is referred to as PLS2. ${ }^{44}$ PLS builds new latent variables (LV) that will define the model complexity, analogously to PCs in a PCA model. Many extensions of PLS can be found in the literature. ${ }^{45-47}$ For example, OPLS is when orthogonal signal correction (OSC) $)^{48}$ pre-processing technique is adapted to each iteration of PLS to remove from the $\mathbf{X}$ matrix the non-correlated information with y. ${ }^{49}$ Another example is sPLS where sparse methods are applied as variable selection steps. This technique uses a penalty term to set to zero uninformative variables in the regression vector. ${ }^{45,50-52}$ Moreover, PLS and its extensions can be adapted to classification purposes, such as PLS-DA (partial least squares-discriminant analysis), as discussed in "2.3. Supervised classification" sub-section.

Classical least squares (CLS) is another regression technique which is based on Beer-Lambert's law for multiple analytes. The spectroscopic data ( $\mathbf{X}$ matrix $)$ can be described as a result of the contributions $(\mathbf{C})$ of each analyte represented by their respective spectral profiles $\left(\mathbf{S}^{\mathrm{T}}\right)$. CLS can be a very powerful tool for pharmaceutical problems, for instance, when the total number of compounds and spectral profiles are well-known. However, this technique is not appropriate when the total number of analytes present in the samples is unknown, nor with complex samples, ${ }^{53}$ as in the majority of forensic problems. Alternatively, resolution techniques are more appropriate in these cases, as it is shown in "2.4. Resolution" sub-section.

\subsubsection{Validation and figures of merit}

Representative samples are at the core of a reliable model, but this is not easily achieved in forensic contexts. When dealing with body fluids, unusual drugs or even a wide variety in the target class, it can be difficult to represent the universe of samples that are being analyzed. Flexible modelling is an important approach to circumvent representativeness problems, from a modeling standpoint, but undoubtedly, a proper validation step can make a big difference.

Each sample set has its own role in model building and, therefore, it is of fundamental importance to report that information when proposing a new methodology. In this review, we address only calibration, validation and prediction sets. A calibration set, also known as training set in a classification context, is usually, although not necessarily, composed by the majority of samples in the dataset. It should contain enough samples to be representative of the dataset. Together with the validation set, it is used to build the model. The validation set can be composed of external samples or the same samples used in the calibration/training set, e.g. when cross-validation or re-sampling techniques are being performed, such as bootstrap for example. ${ }^{54,55}$ This set of samples is used to optimize the model and avoid overfitting problems. When enough samples are available, external validation should be considered, because variability is increased, producing more reliable results. The prediction set must be composed by external samples that were not used for building the model, and it will provide the parameters to assess the models' ability to predict new samples. It is important to emphasize that, in general, the dataset must be split in a way that all the replicates of a particular sample are included in either the calibration or the prediction sets. This is particularly important to ensure that the prediction set is indeed independent from calibration/training. A recent study showed that, in some cases, no statistically significant differences were noted between the two approaches in the final results. ${ }^{56}$ This, however, should not be generalized and, as a rule of thumb, calibration/training and prediction sets should be independent. Notice that independence can have different meanings, in terms of sampling, depending on the target of analysis. In a hypothetical case of classification problem, if one is interested in classifying a given ink entry according to a specific brand and model of pen, then this particular brand and model must be well represented. Different pens should be acquired, and the spectra acquired from all ink entries from an individual pen should belong either to calibration or to prediction set. However, if the target of analysis is to classify a given ink entry according with an individual pen, the independence is related to each entry, and all spectra from a specific entry should belong to either the calibration set or the prediction set.

For calibration purposes, the main parameters being evaluated to assess the final performance of the model include the coefficient of determination $\left(\mathrm{R}^{2}\right)$, the root mean square error (RMSE) and bias. $\mathrm{R}^{2}$ represents the amount of variability from the dataset which is explained by the model and ranges from 0 to 1 (or 100\%). A model with low residual values should have $\mathrm{R}^{2}$ ca. 1 , meaning that the quadratic sum of regression is similar to the total quadratic sum (equation 1). RMSE measures the global error in the model and can be calculated for the calibration (RMSEC), validation (RMSEV or RMSECV, in case of cross-validation) and prediction (RMSEP) sets, as shown in equation 2. Bias indicate systematic errors, according to equation 3 .

$\mathrm{R}^{2}=\frac{\sum\left(\hat{\mathrm{y}}_{\mathrm{i}}-\overline{\mathrm{y}}\right)^{2}}{\sum\left(\mathrm{y}_{\mathrm{i}}-\overline{\mathrm{y}}\right)^{2}}$ 
RMSE $=\sqrt{\frac{\sum\left(\mathrm{y}_{\mathrm{i}}-\hat{\mathrm{y}}_{\mathrm{i}}\right)^{2}}{\mathrm{~N}}}$

bias $=\frac{\sum\left(\mathrm{y}_{\mathrm{i}}-\hat{\mathrm{y}}_{\mathrm{i}}\right)}{\mathrm{N}}$

where $\hat{y}_{i}, \bar{y}$ and $y_{i}$ are the predicted, the mean and the $i^{\text {th }}$ value of the dependent variable; and $\mathrm{N}$ is the number of samples in the validation or prediction sets or the degrees of freedom in the calibration set.

At this point, it is important to emphasize that bias estimation should be carried out using prediction samples to provide a significant and reliable result of model performance, avoiding the optimistic result that will probably be provided by calibration samples. In addition, it is important that a proper significance test is performed (such as $t$-test, $F$-tests, among others) to investigate such things as bias or significant differences between models. For further information on this, the reader is encouraged to consult the ASTM recommendation ${ }^{57}$ for practices on multivariate quantitative analysis.

Other important figures of merit related to the performance of calibration models that should be reported are detection limit (LOD), uncertainty and precision. Those quality parameters are crucial for understanding model validity and applicability, especially in real cases, such as forensic scenarios. LOD, for instance, refers to the lowest amount of a given compound that provides an instrumental response that is statistically different from the background, in a given confidence interval. ${ }^{58}$ This confidence interval plays an important role in the definition of the detection limit since it is able to differentiate the LOD from the critical level of the decision limit (LC). The latter defines a minimum quantity needed for identification of the analyte but without a sufficient level of confidence. This is particularly important to differentiate among the evidence of absence, the lack of evidence, and the evidence of presence of a given analyte, as discussed by Olivieri ${ }^{59}$ and Olivieri and Escandar. ${ }^{60}$

Uncertainty measurements are crucial to provide the reliability of a given methodology. Since the calibration parameters are considered infinitely precise, the only source of errors should be related to spectral acquisition and measurements. Three main sources of errors can affect the uncertainty of the model: (i) spectral measurements from unknown samples; (ii) spectral measurements from calibration samples; and (iii) measurements from the reference values (concentration or a parameter of interest).

When reporting a model result, it is important to provide information regarding the concordance between two or more results. These precision estimations are usually reported in terms of standard deviation and can include repeatability, reproducibility and intermediate precision measurements. For more detail and comprehensive reading on the concept of figures of merit and its role in calibration models, the following references are suggested..$^{59,61}$

In the forensic scenario, those figures of merit are seldom reported, but extremely valuable to ensure the analyst of the reliability of the model that is being proposed. In fact, it is the lack of reliability in forensic results that allow flawed analysis and wrong convictions to occur, a subject of discussion for some years. ${ }^{62}$

\subsection{Supervised classification}

Classification is the process of assigning an individual sample to a specific class or category with similar features. ${ }^{63}$ Classification techniques differ in several ways and can be categorized according to the linearity of the method employed to define the decision boundary, the probabilistic nature of the technique (parametric or non-parametric) and the nature of discrimination. In respect to the latter, a fundamental difference must be made among classification techniques, that is, class-modeling and discriminant analysis. ${ }^{63,64}$ But first, it is of utmost importance to define the problem from the analytical, the forensic and the chemometric point of view before applying any classification technique. From the analytical point of view, decisions should be made according to the analytical technique employed, the type of sample and the target of analysis. A discussion of this can be found in "3. Raman Spectroscopy" and "4. Infrared Spectroscopy" sections.

From the application point of view, it is important to define whether a type of error cannot be tolerated. For example, the analysis of an unknown sample should provide positive or negative responses for a given class and consequently, two types of errors arise, type I and type II. Type I correspond to the false positive cases, where a negative sample is assigned as positive to the analyzed class. On the other hand, type II errors correspond to the case where a positive sample is assigned as negative to the analyzed class, ${ }^{27}$ i.e., false negatives. This is especially important because the final model must compromise between the two types of errors and the purpose of the classification. Equally important is the decision of which type of error is more critical and this will depend on the target and protocol of analysis followed. It is also necessary to address the mathematical point of view, by defining how many target classes are being evaluated and whether hierarchy is important in the classification.

\subsubsection{Class modeling}

Class-modeling consists of a set of techniques in which 
the classification is focused on the similarities of samples that belong to the same class rather than differences between classes. Therefore, the mathematical boundary of a given class will (or should) be built based only on the samples that belong to the target class. ${ }^{63-65}$ This is a fundamental difference between class-modeling and discriminant analysis and it has been discussed in recent years for food authenticity. ${ }^{65-68}$ Those techniques are especially interesting when the non-class-belonging samples do not share similar characteristics, i.e., there are a variety of samples that do not belong to the target class.

Discriminant analysis, however, is the one more frequently employed. According to Oliveri and Downey, ${ }^{69}$ this is due to the fact that discriminant analysis was developed first, it is available most of commercial software and results are apparently better than with class-modeling. Nevertheless, there are many powerful class-modeling techniques that need to be considered for forensic purposes, such as unequal class model (UNEQ) ${ }^{70}$ potential function methods (PFM) ${ }^{71}$ partial least squares density modeling (PLS-DM), ${ }^{72}$ and one-class partial least squares (OCPLS) ${ }^{73}$ However, to our knowledge, the only class-modeling technique employed for forensic purposes so far, with exception of applications in counterfeit food, is soft independent modelling of class analogy (SIMCA). This was also observed by Kumar and Sharma. ${ }^{16}$ The authors of the present review feel that class-modeling techniques have been underrated in forensic applications.

$\mathrm{SIMCA}^{74}$ is a classification technique based on PCA where each class of interest is independently modelled according to the class-belonging samples. In this case, PCA is used to model and describe class variance independently. The number of relevant PCs can be defined to each class during the validation process. Geometrically speaking, each class will be defined by a region in the hyperspace of PCs, i.e., a subspace defined by the number of relevant PCs. The classification process is distance-based and different methods can be adopted for class assignment. The criterion for decision-making in the past was based on residuals followed by an $\mathrm{F}$-test ${ }^{74}$ but, more recently, different decision criteria have been proposed in the literature, ${ }^{75-80}$ such as the Mahalanobis distance in the score space. Although the independent-modeling characteristic can be a positive feature of SIMCA models, it can also be a disadvantage in the sense that the modeling process does not seek for a specific direction in which the class separation is maximized ${ }^{64}$ Consequently, this can lead to classification errors or unassigned samples.

Modification on SIMCA algorithm has been recently made by using score and orthogonal distances calculated from a particular dataset to stablish a cutoff limit of acceptance for the target class. This modified version of SIMCA model is known as data driven-SIMCA (DD-SIMCA) and it provides significant advantages when modeling contaminated datasets, taking into account outliers and extreme samples (not outliers), as these play important roles during model building and the decision boundary. ${ }^{80-82}$

\subsubsection{Discriminant analysis}

Contrary to class-modelling techniques where regions in the hyperspace are defined for each class, discriminant analysis divides the hyperspace into as many regions as classes analyzed. This generates an important aspect about classification by discriminant analysis: a sample will always be classified in one class. The literature reports other approaches, in which a step for outlier detection for discriminant analysis allows the analyst to eliminate samples, generating unassigned samples. ${ }^{83,84}$ Regarding class-modeling techniques, samples can be assigned simultaneously to several classes or to none. Such conceptual differences are extremely important in forensic contexts and must be taken into consideration, depending on the problem at hand.

Furthermore, discriminant analysis has the advantage that the models usually seek the maximum separation between the different classes thus generally providing better results. In this case, it is important that non-target analysis is well represented, since class boundaries will be defined based on both class-belonging and non-class-belonging samples. The function that defines class boundaries and projections will depend upon the technique, which, in its turn, can generate linear or non-linear boundaries. The most popular discriminant analysis techniques used in forensic applications include partial least squaresdiscriminant analysis (PLS-DA) (and some of its extensions such as sparse, recursive, and orthogonal, among other modifications), linear and quadratic discriminant analysis (LDA and QDA, respectively) and support vector machinesdiscriminant analysis (SVM-DA).

PLS-DA is essentially PLS regression for discrimination purposes. ${ }^{85}$ In this case, instead of the $\mathbf{Y}$ matrix (or $\mathbf{y}$ vector), it consists of quantitative values of a parameter that will be replaced by numerical representations for a class membership. Usually, a binary code is employed, such as (1) for class-belonging and (0) for non-class-belonging samples, and the value for the predicted class, either $\hat{\mathbf{y}}$ or $\hat{\mathbf{Y}}$, will be a real value $\left(\hat{\mathbf{y}}_{\mathbf{i}} \in \mathbb{R}\right)$. Additionally, a threshold must be defined to assign a sample to a given class.

$\mathrm{LDA}^{86}$ is one of the most popular discrimination techniques employed. The goal is to find directions, known as discriminant functions, in which the variance between different classes is maximized and the variance 
within samples from the same class is minimized. A linear boundary is defined based on a discriminant rule that optimizes the misclassification rate.$^{64,87}$ By building an LDA model, it must be assumed that all classes are normally distributed and have the same variance. However, data can be more complex and classes often show different variance. In these cases, quadratic boundaries such as QDA are preferred. ${ }^{63}$ It is important to mention that LDA and QDA methods employ the inverse of the covariance matrix, which is rather difficult to calculate; thus, the number of samples must be higher than the number of variables. Therefore, variable selection techniques ${ }^{46,88-90}$ or methods for dimensionality reduction such as PCA should be employed beforehand.

SVM-DA has become a popular classification technique in different fields of science, including forensics, although it can also be used for regression purposes. ${ }^{91}$ One reason for this is the fact that it handles linear and non-linear data. The simplest approach is linear separation, where samples from different classes are selected to determine the decision function and establish the class boundary. As such, those particular samples located right at the class border are called support vectors (SVs) and the decision function (or hyperplane) will be defined in such a way that it is equidistant from the extreme samples of each class. It is also important to emphasize that the hyperplane should be defined so that the difference between the extreme samples is maximized. Many authors argue that employing SVM technique can be risky in linear problems due to the possibility for overfitting. ${ }^{92}$ In more complex cases, it should be adapted with some additional steps incorporated. Thus, instead of building the decision function in the input space (e.g. original variables and PCA score space), it should employ a feature map to expand the space dimension in such a way that classes are linearly separated. In this case, the linear function in the spanned space will provide a non-linear boundary in the input space. ${ }^{93}$ The feature mapping step is achieved by means of feature functions $\phi(x)$ that can be found by kernel methods. The most common kernel functions employed in SVM models are radial function basis (RBF), polynomial function (PF) and sigmoidal function (SF). Moreover, a penalty term can be employed to control the complexity of the models and avoid problems of overfitting. ${ }^{92}$

\subsubsection{Figures of merit}

The figures of merit used to assess the performance of classification models are rather different from calibration. For interesting reading, the theory of each technique, consider Ballabio et al. ${ }^{94}$ López et al. ${ }^{95}$ and Ellison and Fearn. ${ }^{96}$ The main ones used in forensic contexts are briefly discussed below.
After building a model and assigning samples to classes, a contingency table, or confusion matrix, as it is most commonly known, should be provided. This table shows the relationships between the actual and the predicted result for each class, i.e., the number of correct and incorrect classification of samples in a certain class. It is important to mention that the figures of merit can be class-specific or global. The class-specific include sensitivity of a g-class $\left(\mathrm{Sn}_{\mathrm{g}}\right)$, which relates to the true positive rate, i.e., gives the ratio between g-class samples actually predicted in $\mathrm{g}\left(\mathrm{c}_{\mathrm{gg}}\right)$ and the total number of samples from g-class $\left(\mathrm{n}_{\mathrm{g}}\right)$, as described in equation 4. Consequently, sensitivity is also a measurement for false negative cases. On the other hand, specificity of a given g-class $\left(\mathrm{Sp}_{\mathrm{g}}\right)$ relates to the true negative rate and is calculated by the ratio between the number of samples that do not belong to g-class which were not assigned to $g$ and the total number of samples that do not belong to $\mathrm{g}$, as can be noticed in equation 5 .

$$
\begin{aligned}
& \mathrm{Sn}_{\mathrm{g}}=\frac{\mathrm{c}_{\mathrm{gg}}}{\mathrm{n}_{\mathrm{g}}} \\
& \mathrm{Sp}_{\mathrm{g}}=\frac{\sum_{\mathrm{k}=1, \mathrm{k} \neq \mathrm{g}}^{\mathrm{G}}\left(\mathrm{n}_{\mathrm{k}}-\mathrm{c}_{\mathrm{kg}}\right)}{\mathrm{n}-\mathrm{n}_{\mathrm{g}}}
\end{aligned}
$$

where $\mathrm{G}$ stands for the number of classes; $\mathrm{k}$ to the $\mathrm{k}^{\text {th }}$-class; $\mathrm{n}$ the total number of samples; and $\mathrm{c}_{\mathrm{kg}}$ the number of samples from $\mathrm{k}$ predicted as $\mathrm{g}$.

Accuracy and non-error rate (NER) are global parameters and should be carefully interpreted because class-specific information cannot be assessed and usually does not take into account unbalanced classes. Accuracy relates to the overall correct classification ratio and NER to the average sensitivity (equations 6 and 7), they both range from 0 to 1 (or 100\%), where 1 means perfect classification and 0 means that the model is not adequate. Parameters such as accordance (ACC) and concordance (CON) can also be found, which is an attempt to address repeatability and intermediate precision of qualitative results, respectively. ${ }^{96}$

Accuracy $=\frac{\sum \mathrm{c}_{\mathrm{gg}}}{\mathrm{n}}$

$\mathrm{NER}=\frac{\sum \mathrm{Sn}_{\mathrm{g}}}{\mathrm{G}}$

\subsection{Resolution}

Resolution techniques can be extremely powerful in forensic chemistry, particularly multivariate curve resolutionalternating least squares (MCR-ALS) due to its high 
flexibility during model building. MCR-ALS belongs to a class of techniques developed to solve mixture problems, decomposing the observed data into pure chemical profiles and their respective contributions. ${ }^{97} \mathrm{MCR}-\mathrm{ALS}$ relies on the Beer-Lambert law in which an iterative algorithm is designed to seek for possible solutions for equation 8 .

$$
\mathbf{X}=\mathbf{C S}^{\mathrm{T}}+\mathbf{E}
$$

In order to do this, initial estimates of spectral $\left(\mathbf{S}^{\mathrm{T}}\right)$ or concentration $(\mathbf{C})$ profiles are needed (E refers to residuals). They can be obtained in different ways, for example, providing the known profiles or using methods like evolving factor analysis (EFA) $)^{98}$ and SIMPLISMA. ${ }^{99}$ When spectral profiles are provided, they can be used to estimate the concentration profiles to fit the observed data; and once the concentration profiles are obtained, the spectral profiles can be optimized to best fit the dataset in an iterative way, by employing the ALS algorithm. The iteration process runs until a convergence criterion is reached.

It is important to be aware that rotational and intensity ambiguities are inherent to MCR-ALS, ${ }^{100}$ and several solutions that are mathematically acceptable can be obtained. To circumvent this issue, different constraints can be applied to narrow down the range of possible solutions and achieve one with a chemical meaning. Non negativity, unimodality, closure, and selectivity are some of the main constraints that can be found in the literature, ${ }^{100-102}$ although new developments, especially in image analysis, are also described. ${ }^{103-106}$

The most interesting feature about MCR-ALS in forensic chemistry applications is the ability to find the analyte or the target in different, and often unpredictable, "chemical contexts". In some forensic scenarios, the target of analysis is usually well known and spectral profiles for initial estimations are already available. In other cases, however, such as illicit drugs, the target analyte can be mixed with a variety of other compounds (adulterants, diluents, etc.). This is also true in complex biological matrices (blood, saliva, urine, etc.), which poses an analytical challenge. Another advantage of MCR-ALS is the possibility of employing augmented matrices with relevant information to improve the final solution. This can be done using different strategies, for example, by concatenating spectra from different techniques in the spectral direction (column-wise) or spectra from other samples (row-wise), like reference spectra.

\section{Raman Spectroscopy}

Raman spectroscopy is based on the inelastic scattering of light when a monochromatic laser beam strikes matter. The scattered light has a different frequency from the laser frequency and comes from vibrations that caused a change in the polarization of the electron cloud of the molecule. Thus, the relative frequency is characteristic of the molecular vibrations and the chemical structure. Raman has particular advantages in forensic chemistry. It can analyze a wide range of samples, independent of their chemical state and without the need for sample preparation. This is most advantageous for in loco analysis. Raman is nondestructive due to the low-intensity laser powers employed, necessary to maintain trace integrity. The experimental parameters are very few, when compared to other analytical techniques, and the process requires only a few seconds to obtain spectral information with sufficient signal-to-noise ratio, which makes it a relatively simple and rapid technique. Spectra are highly informative, covering the region from 4000 to $50 \mathrm{~cm}^{-1}$ in a single measurement, and can have many sharp peaks.

In terms of instrumentation, Raman is very versatile. The finely focused laser beam allows for the analysis of small areas. Spatial resolutions of $1 \mu \mathrm{m}$ or less are possible when the equipment is coupled with a powerful microscope. Most modern instruments have automatic sampling stages for two/three dimension-spectroscopic mapping and imaging applications. This is particularly useful for obtaining spatial information of heterogenic samples. Other instruments have an optic fiber probe for remote and standoff applications. A variety of lasers are available, which is useful for complementary analysis of mixtures and for obtaining higher discriminating power. Additionally, the laser beam has a shallow penetration depth, which makes it attractive for surface analysis.

Some limitations of this technique include fluorescence, from impurities or the sample itself, which appear as broad, curved and intense signals that mask the naturally weaker Raman peaks. Moreover, the laser beam can be too intense for a small focus area and cause heating and burning of the sample. Regulation of the laser power can avoid this. There are a few countermeasures to the fluorescence problem. One solution is to use lasers with longer excitation wavelengths that have less energy. However, the most effective approach is performing surface-enhanced Raman spectroscopy (SERS) which involves close contact of the sample with metallic nanosubstrates (typically silver or gold). It is known that the surface plasmon of the nanosubstrate-analyte amplifies the intensity of the Raman signal up to $10^{3}$ to $10^{6}$ orders of magnitude and simultaneously quenches fluorescence. Recent applications of SERS in forensic science have been reviewed elsewhere, ${ }^{107}$ the use of chemometrics in combination with SERS applications, however, is still 
rare. Besides SERS, spatially offset Raman spectroscopy (SORS) and time-resolved Raman spectroscopy (TRRS) have shown potential for identification of drugs and explosives in opaque containers. ${ }^{108-110}$

Raman spectroscopy has advantages and drawbacks compared with infrared spectroscopy. The main advantages are that water has a minimum or no spectral signal, which enables analysis of aqueous solutions and samples with high water content without major interferences. In addition, the analysis of inorganic materials is easier and samples can be analyzed through transparent glass or polymer packaging. In spite of this, Raman is not as well established in forensic laboratories as infrared spectroscopy. This may be due to the fact that, in general, forensic experts are not familiar with the technique, the instrumentation is more expensive and it is not as accessible as infrared spectroscopy.

\section{Infrared Spectroscopy}

Infrared (IR) spectroscopy is based on molecular absorption of energy which causes vibrational and rotational transitions. IR comprises the spectral ranges between 12,800 to $10 \mathrm{~cm}^{-1}$, among which three different regions can be identified: the near infrared (NIR) from 12800 to $4000 \mathrm{~cm}^{-1}$, the middle infrared (MIR) from 4000 to $200 \mathrm{~cm}^{-1}$, and the far infrared (FIR) from 200 to $10 \mathrm{~cm}^{-1}$. MIR and NIR in particular are have been widely employed in forensic applications, providing information about fundamental transitions, overtones and combination bands.

The wide acceptance of IR to deal with forensic problems is due to the high versatility of the technique to analyze liquid, solid or gaseous samples with minimum or no sample pretreatment, in a non-destructive and often noninvasive way. While the MIR region provides information about functional groups, NIR spectra are more complex and often requires statistical techniques to unravel the chemical information. ${ }^{111}$ Despite this complexity, in terms of instrumentation, NIR is simple, low-cost, robust and easily miniaturized, ${ }^{112,113}$ particularly useful for analysis in loco.

Additionally, some NIR instruments enable image acquisitions of considerably large areas very rapidly. ${ }^{114}$ Also known as hyperspectral images (HSI), this type of data provides the chemical information about a compound as well as its spatial distribution. For a more detailed discussion of HSI, the reader should consider the following references. ${ }^{111-113,115-119}$

The simplicity of instrumentation and speed of spectral acquisition of IR instruments is a great advantage over Raman, particularly for the analysis of large sampling areas. However, water gives intense and broad absorption peaks that can overlap the contributions from important compounds. This may be an advantage, however, if the purpose is to determine the water content of materials. In addition, IR spectra do not suffer interference from fluorescence, which is a common problem in Raman spectroscopy.

\section{Questioned Documents and Currency}

Forensic document examination is of vital importance in any forensic laboratory. Forensic scientists examine and compare handwriting, typewriting, printing processes, inks, paper and other document characteristics that can attest to the authenticity or fraudulent nature of identity, security or any sort of fraudulent paper use. Ink or paper differentiation, altered numbers and obliterations are among the cases most often encountered. Other more complex cases include determining the order of crossing ink lines and aging estimation. A comprehensive review of the analytical techniques used for examination of questioned documents has been published elsewhere. ${ }^{120}$

\subsection{Raman spectroscopy in document analysis}

Raman spectroscopy is very advantageous for ink analysis of questioned documents because paper has a negligible signal compared to inks. Hoehse et al. ${ }^{121}$ analyzed ten blue and black ink samples in paper using a confocal Raman instrument and laser-induced breakdown spectroscopy (LIBS). They applied PCA and, for classification purposes, SIMCA, PLS-DA, and SVM-DA models were built and compared. PCA showed interesting cluster structures, with relative confusion of some pairs of samples. SIMCA showed similar results as PCA, as expected. All ink pairs were separated by the PLS-DA model. When the Raman and LIBS spectra were fused, an improvement was observed in PCA, SIMCA and PLS-DA performances. SVM-DA yielded high classification rates, but these were not improved by the data fusion approach. Some authors have argued that SVM-DA can be employed as a screening technique due to its reduced calculation time when compared to PCA. However, this depends highly on the algorithms employed by the software for PCA and SVM-DA optimization. In any case, unsupervised techniques remain simpler and preferable for screening analysis.

Poppi and co-workers ${ }^{122,123}$ have published two studies on this. In the first, ${ }^{122}$ they analyzed genuine, counterfeit and homemade counterfeit banknotes using a confocal Raman instrument (laser excitation $785 \mathrm{~nm}$ ). After band identification of the main pigments, exploratory PCA 
analysis was performed which showed a clear differentiation between authentic and counterfeit banknotes, owing to the different printing mechanisms used in the manufacturing process. Therefore, for PLS-DA classification, data were divided according to the printing type (calcographic for genuine bills, laser and inkjet for counterfeits). Two calibration models were constructed, the first to classify real and counterfeit samples and the second to classify the counterfeits as laser or inkjet. The threshold used to split the classes varied according to the least probability of false classifications in the calibration model. They used the bootstrap residual re-sampling technique to estimate the confidence interval of prediction for PLS-DA models. Regarding the first model, all authentic samples were correctly classified. Regarding the second model, some laser printer samples were incorrectly classified as inkjet samples, which the authors explained was due to either noisy spectra or mixture of inks that was not under consideration. In another study, ${ }^{123}$ simulated samples of forgery by adding text were made with 7 ballpoint pens on white paper and two different bank checks. The number ' 30 ' was forged to ' 80 ' with different pens and the Raman image was acquired. Data were treated with MF-ICA, after estimating the number of independent components by singular value decomposition (SVD). A high percentage of explained variance was obtained for the models and the correlation between the Raman spectrum and the initial estimates of the inks was higher than 0.85 . The authors observed that a high number of components (up to 5 or 6) were necessary to account for contributions of physical variations on the paper surface or detect products originating from the different manufacturing of paper. Finally, a distribution map related to each ink enabled the simulated forgery to be observed.

Braz et al. ${ }^{124,125}$ published two studies on the matter. In the first, ${ }^{124}$ they investigated the Raman spectral variability of 190 blue pens of different ink types, brands, models and batches. A confocal Raman instrument (laser excitation $532 \mathrm{~nm}$ ) was used. After band assignment and identification of the main dyes or pigments, PCA models were built to visualize the differences among the samples. PCA models were able to show different batches and models of pens. The authors suggested that the changes have been introduced in the chemical formulae of the pens over the years which means that the forensic examiner must keep up with these market tendencies. In the second study, ${ }^{125}$ they analyzed crossings between different types of blue pens, written on different papers, and with different times separating the application of the two ink lines. They used a Raman imaging instrument (laser excitation $532 \mathrm{~nm}$ ) and MCR-ALS with two components for resolving the spectral profiles of the inks at the crossing area. The MCR-ALS enabled visual determination of the order of crossings in more than $60 \%$ of the cases. MCR-ALS is an adequate technique for this application because initial estimates for both inks can be obtained directly from the ink lines. Techniques such as ICA can also be used as an alternative for these cases.

Borba et al. ${ }^{126}$ analyzed obliterations and cases of crossing lines made with ballpoint pens using Raman imaging at different depths in the sample (laser excitation $532 \mathrm{~nm}$ ). MCR-ALS and a local rank constraint with fixed size image window-evolving factor analysis (FSIW-EFA) was used to treat the data. The FSIW-EFA algorithm performs local singular value decompositions in a $2 \times 2$ pixel window in all the data to identify which components are present in each pixel. Instead of a single threshold value, the authors used a threshold band, which allowed determination of the consistency of the rank information. The authors claimed that this is particularly important when inks have spectral similarity. The distribution maps and pixel concentrations in the images at different depths allowed for satisfactory identification of the order of crossings and the cases of obliteration.

Buzzini et al. ${ }^{127}$ analyzed inkjet printed samples by Raman using two laser excitations, 532 and $785 \mathrm{~nm}$, to reveal Raman signals which had been undetected by one of the lasers due to resonance effects. PCA was used for more objective discrimination of the inks involved, although the authors claimed that no improvements were achieved from a visual comparison of the spectra. They also mentioned that spectral pre-processing was not optimized and this might have influenced the PCA results.

\subsection{Infrared spectroscopy}

IR spectroscopy has the potential for identifying the chemical structure of the functional groups present in inks, colorants and resins. Differently from Raman, IR can be particularly useful for chemical analysis of paper. This can be an advantage, when the focus is paper discrimination, but can also be a disadvantage, when the focus is on ink analysis because deeper radiation penetration, especially NIR, will result in interfering signals.

Silva et al. ${ }^{128,129}$ published two studies, where in the first, ${ }^{128}$ they used attenuated total reflectance-Fourier transform infrared spectroscopy (ATR-FTIR) and LDA for classification of blue pen inks according to their types and brands. They analyzed written ink spots made with 100 pens (ballpoint, rollerball and gel) from ten different brands on three types of paper. They used three approaches for the selection of spectral variables: genetic algorithm (GA), stepwise formulation (SW) and the successive projections 
algorithm (SPA). Two LDA models were constructed using blue pen ink spectra obtained from one brand on sulfite paper, one for classification according to the type of pen and another according to the brand of pen used. Three prediction sets were employed using the spectra of blue inks used on the three papers. The influence of paper was evaluated on the model predictions, when different brands were considered. Even though, high classification rates were achieved in all cases, results for recycled paper have shown lower correct classification rates. Overall, LDA-SPA and LDA-GA showed better performance than LDA-SW models, which was significantly better than the results published by one of the first studies on the same matter. ${ }^{130}$ In the second study, ${ }^{129}$ they demonstrated the potential of NIR imaging for detecting number alterations on bank checks, revealing obliterated texts, and determining the order of crossing lines. They used black pens of different ink types and toners to produce the samples. The score images of PCA and MCR-ALS were used to elucidate the cases of text addition and obliteration. Both MCR-ALS and PLSDA (as a variable selection tool) were used to determine the order of crossing lines. The obliterated text was successfully identified in $43 \%$ of the cases using all methods. Text additions were successfully identified in $82 \%$ of the cases and the order of crossings between pens and toners were successfully identified in $85 \%$ of the cases. In both cases, the influence of paper is evident, but the results still show the potential of NIR spectroscopy for ink analysis.

Pereira et al. ${ }^{131}$ evaluated and compared the use of NIR and MIR imaging and two unsupervised pattern recognition techniques (PCA and PP) for the identification of document forgery by means of alterations and additions. Different black ink pens were employed to produce a total of 120 pairs of samples for discrimination. They performed blind testing with 30 different simulated cases of either genuine or altered/added numbers on white or bank check paper. Initial pre-treatment based on the region of interest (ROI) selection and PCA histograms was necessary to remove the interfering paper contributions from the ink. Overall, PP analysis showed better results than PCA, either using MIR (97.5 and $87.5 \%$, respectively) or NIR (83.3 and $76.7 \%$, respectively) imaging. However, their complementary use enabled identification of up to $90 \%$ of the blind samples, which indicates the potential for use of the methodology proposed.

Sharma and Kumar ${ }^{132}$ compared the performance of high-performance thin layer chromatography (HPTLC) and ATR-FTIR to discriminate among 57 blue ballpoint line strokes. Two approaches were employed for ATR-FTIR: a univariate, by visual examination of the spectra (number and position of peaks) and a multivariate approach, by using PCA and Varimax rotation, which is a rotation tool that changes the directions of the PCs vectors for new linear combinations of LVs that maximize the variance of the loadings. The multivariate approach enabled identification of $99.69 \%$ of the samples, which was higher when compared to both the univariate approach (97.93\%) and HPTLC $(93.80 \%)$.

Lee et al..$^{56,133}$ recently published two relevant studies that evaluated the appropriate use of chemometrics in classification of ink lines in questioned documents. In the first ${ }^{56}$ they discussed whether ink strokes made with the same pen should be considered replicates or independent samples for classification purposes and the best way for splitting the dataset into training and test sets for external validation. For this, they analyzed 1361 strokes made by 273 blue gel pen inks from ten different brands and from 23 models using ATR-FTIR. The data set was split into two: set IP (individual pen), where all the strokes from the same pen were included in either the training or validation sets; and set NIP (ink entry of an individual pen), where strokes from the same pen were included in both the training and validation sets. PLS-DA models were built for classification according to the brand and model of pens. Although the authors expected that the inclusion of replicates of the same pen (set NIP) would make the set perform much better, results were in fact similar in terms of accuracy, stability and fitting. The authors claim that, since the goal of this kind of classification is to determine the brand or model of a questioned stroke rather than the specific pen employed, the use of replicates in both the training and validation sets would provide a more realistic predictive performance, although in realistic scenario this is not a possibility. In the second study, ${ }^{133}$ they used the same dataset to evaluate the effect of nine different preprocessing techniques on the prediction performance of the PLS-DA models. The main conclusions are relatively obvious for expert users of IR spectroscopy, that is, models perform better when slope-correcting methods such as asymmetric least squares and normalization to sum, are used and when regions with fewer contributions from paper substrate are chosen. These contributions are fundamental for establishing the use of chemometrics techniques within the forensic sciences because they lead to a discussion of theoretical issues focused on a particular forensic problem.

Asri et al..$^{134}$ evaluated and compared three strategies for the identification of the source of eighteen red gel pen inks deposited on a simulated threatening note, using Raman and ATR-FTIR. Neither visual comparison of the spectra nor Pearson's product moment correlation (PPMC) coefficient (r) calculated among all possible pairs of samples were able to determine the actual source of the unknown ink. 
However, the PCA scores plot performed using both Raman and FTIR datasets successfully linked the unknown ink to the actual pen used to write the simulated threatening note.

Materazzi et al. ${ }^{135}$ investigated the use of reflectance NIR and PCA to discriminate among ten black toners from four brands printed on white paper. They evaluated several parameters: different papers, the influence of the printing machine and the printing process, for which they used four printers, the use of full or a low percentage of ink in the cartridge, and replicas prepared on different days. The PCA scores plots showed different clusters according to each brand of toner. The analysis, on the other hand, did not show significant dependence on the printing process nor were the results affected by the type of paper and percentage of ink in the cartridge used.

Kumar and Sharma ${ }^{136}$ used ATR-FTIR associated with PCA and Varimax rotation for characterization and discrimination of twenty-four papers of different brands. Three spectral regions were chosen for qualitative and multivariate examination, 400-2000, 2000-4000 and $400-4000 \mathrm{~cm}^{-1}$. Results showed that $97.83 \%$ of the paper samples were identified based on qualitative spectral features; the chemometric approach obtained $99.64 \%$ discrimination, particularly when the spectral range of 2000-4000 $\mathrm{cm}^{-1}$ was employed.

Two studies ${ }^{137,138}$ have been published that investigate the aging of paper in documents. In the first, Silva et al. ${ }^{137}$ used ATR-FTIR to analyze naturally-aged paper documents from 15 different years (between 1985 and 2012), considering five documents per year on five different sheets of paper. Two datasets were built and two filters (generalized least squares weighting, GLSW, and OSC) and sPLS were applied and compared for reducing the variability among papers of the same period. Results showed improvements in attenuating the variability among documents with the same age using all methods. Values of approximately 4 years were obtained for RMSECV and RMSEP, but variable importance in projection (VIP) scores showed that spectral regions associated with inorganic compounds were influencing the models. The OSC filter was more suitable for the analyzed dataset. The authors emphasized the importance of sample sets, showing how the results can be affected by the manner the datasets were built. This opened a discussion on the advantages and drawbacks of chemometric techniques applied to document dating. In the second study, Risoluti et al. ${ }^{138}$ used a miniature NIR spectrometer and PCA to characterize and investigate dating of paper. Two sets of books and sheets were used: books with publication dates from $18^{\text {th }}$ century (1700) to the $20^{\text {th }}$ century (1952), which had been well preserved from the environmental conditions; and paper sheets from the $16^{\text {th }}$ century $(1500)$ to the $21^{\text {st }}$ century (2016), which had been exposed to degradation. The PCA score plots showed some variations among the samples based on chemical differences on the surface. The authors have suggested that this may be related to the period when the documents were made.

Oliveira et al. ${ }^{139}$ used SIMCA and LDA-SPA to classify 100 authentic and 227 counterfeit Brazilian Real bank notes using a portable NIR. They analyzed seven different regions on the banknotes, including two with fluorescent ink, one over the watermark, three from the intaglio printing and one over the serial numbers. They built models for authentic and counterfeit banknotes. PCA showed differences between the authentic and counterfeit samples, particularly in the intaglio printing areas analyzed. Both SIMCA and LDA-SPA models correctly classified all authentic banknotes. The authors argued that LDA-SPA is simpler and the variable selection step has the advantage of removing noise and nonlinearity. However, in these cases where the target class is highly controlled and easily modeled, other class-modeling techniques could also be explored. In any case, both classification techniques showed high potential for routine forensic applications.

Table 1 shows a resume of the research works and chemometric techniques for questioned documents.

As depicted, confocal Raman was preferred for discrimination of inks and HSI for larger areas like crossings, document alterations and currency. The $532 \mathrm{~nm}$ laser excitation was used for most applications. PCA was preferred for preliminary analysis, different classification techniques (SIMCA, PLS-DA, SVM-DA, ICA) were used for discrimination issues and the resolution technique MCR-ALS for HSI applications. ATR-FTIR was preferred for discrimination of inks and paper and HSI in the NIR and MIR for crossings, document alterations and currency. PCA was used alone or with another exploratory technique like PP and Varimax rotation. Classification techniques (LDA, PLS-DA) were preferred for discrimination issues and ATR-FTIR data analysis. The calibration technique PLS was used for investigations of aging.

\section{Gunshot Residues (GSR)}

GSR are a set of burned and unburned particles from the projectile and the gun discharge. ${ }^{140}$ From a forensic perspective, the identification of GSR is important for estimating shooting distance, identifying bullet holes, connecting the suspect/weapon to a crime, confirming that a weapon has been fired and confirming a suicide hypothesis. GSR can be inorganic, composed of heavy metals, inorganic salts, and graphite (a.k.a. IGSR) from the 
Table 1. Resume of techniques for questioned documents and currency

\begin{tabular}{|c|c|c|c|}
\hline Analytical technique & Chemometric technique & Sample & Reference \\
\hline Confocal Raman $(488,633,785 \mathrm{~nm})$, LIBS & PCA, ${ }^{a}$ SIMCA, ${ }^{b}$ PLS-DA, ${ }^{b}$ SVM-DA ${ }^{b}$ & blue and black inks on paper & 121 \\
\hline Confocal Raman (785 nm) & PCA, ${ }^{a}$ PLS-DA ${ }^{b}$ & authentic and counterfeit banknotes & 122 \\
\hline HSI-Raman (785 nm) & $\mathrm{MF} \mathrm{ICA}^{\mathrm{a}}$ & number alterations paper and bank checks & 123 \\
\hline Confocal Raman (532 nm) & $\mathrm{PCA}^{\mathrm{a}}$ & ink on paper & 124 \\
\hline HSI-Raman (532 nm) & MCR-ALS & crossing ink lines (surface analysis) & 125 \\
\hline HSI-Raman (532 nm) & MCR-ALS & crossing ink lines (depth-profiling) & 126 \\
\hline Confocal Raman $(532,785 \mathrm{~nm})$ & $\mathrm{PCA}^{\mathrm{a}}$ & inkjet printed lines & 127 \\
\hline ATR-FTIR & GA-LDA, ${ }^{b}$ SW-LDA, ${ }^{b}$ SPA-LDA ${ }^{b}$ & blue inks on paper & 128 \\
\hline HSI-NIR & PCA, ${ }^{a}$ PLS-DA, ${ }^{b}$ MCR-ALS ${ }^{c}$ & $\begin{array}{l}\text { number alterations on bank checks; obliterated } \\
\text { texts; crossing ink lines }\end{array}$ & 129 \\
\hline HSI-NIR, HSI-MIR & $\mathrm{PCA},{ }^{\mathrm{a}} \mathrm{PP}^{\mathrm{a}}$ & number alterations on paper and bank check & 131 \\
\hline ATR-FTIR, HPLC & $\mathrm{PCA}^{\mathrm{a}}{ }^{\text {Varimax }}$ rotation ${ }^{\mathrm{a}}$ & blue inks on paper & 132 \\
\hline ATR-FTIR & PLS-DA ${ }^{b}$ & blue inks on paper & 56,133 \\
\hline ATR-FTIR, Raman (785 nm) & $\mathrm{PCA}^{\mathrm{a}}$ & red inks on paper & 134 \\
\hline NIR & $\mathrm{PCA}^{\mathrm{a}}$ & toner printed lines & 135 \\
\hline ATR-FTIR & PCA, ${ }^{a}$ Varimax rotation ${ }^{\mathrm{a}}$ & paper & 136 \\
\hline ATR-FTIR & $P L S,{ }^{d}{ }^{2} P L S^{d}$ & aging of paper & 137 \\
\hline NIR (portable) & $\mathrm{PCA}^{\mathrm{a}}$ & aging of paper & 138 \\
\hline NIR (portable) & ${\text { SIMCA },{ }^{\mathrm{b}} \text { SPA-LDA }}^{\mathrm{b}}$ & authentic and counterfeit currency & 139 \\
\hline
\end{tabular}

${ }^{\mathrm{a}}$ Exploratory; ${ }^{\mathrm{b}}$ classification; ${ }^{\mathrm{c}}$ resolution; ${ }^{\mathrm{d}}$ calibration. LIBS: laser-induced breakdown spectroscopy; PCA: principal component analysis; SIMCA: soft independent modeling of class analogy; PLS-DA: partial least squares-discriminant analysis; SVM-DA: support vector machines-discriminant analysis; HSI: hyperspectral images; MF-ICA: mean field independent component analysis; MCR-ALS: multivariate curve resolution-alternating least squares; ATRFTIR: attenuated total reflectance-Fourier transform infrared spectroscopy; GA-LDA: genetic algorithm-linear discriminant analysis; SW-LDA: stepwise formulation-linear discriminant analysis; SPA: successive projection algorithm; NIR: near infrared; PP: projection pursuit; HPLC: high-performance liquid chromatography; sPLS: sparse partial least squares.

primer and the weapon barrel; and organic, composed of explosives, plasticizers, stabilizers, and compounds derived from the propellants (a.k.a. OGSR). These particles are usually visible to the naked eye. ${ }^{141}$ Although the established examinations include elemental and morphology analysis of IGSR using atomic spectroscopy and electron microscopy, researchers have been focusing on the use of OGSR due to the emerging trend of non-toxic lead-free ammunition.

\subsection{Raman spectroscopy}

Raman technique is particularly useful for detecting different metal anions in GSR particles, such as barium, lead and iron oxide, sulfate and carbonate mixtures, and has become an important tool for characterizing the organic components, particularly in lead-free ammunition. However, very few studies have reported the use of chemometrics for GSR analysis using Raman spectroscopy. Lednev and co-workers ${ }^{142-144}$ published three studies on GSR analysis for the discrimination of firearm caliber, using the same confocal Raman instrument (laser excitation
$785 \mathrm{~nm})$. In the first study, ${ }^{142}$ they analyzed GSR particles collected from a cloth, which were fired at from close range $(30 \mathrm{~cm})$ by a $0.38 \mathrm{~mm}$ revolver and a $9 \mathrm{~mm}$ firearm. GSR particles (brown and tan) showed high fluorescence interferences and were discarded; the remainder were divided into two datasets, according to their color. After band assignment of the organic and inorganic compounds of GSR, GLSW preprocessing was applied to attenuate the scattering characteristics of each caliber and PCA was performed, which enabled observation of the separation of samples according to caliber. Then, they employed k-nearest neighbors (k-NN), PLS-DA and SVM-DA for classification. The result was that one spectrum had been misclassified for k-NN and SVM-DA, but PLS-DA gave correct classifications for all samples. Only PLS-DA was tested with a prediction set, providing perfect classification according to caliber. In another study, ${ }^{143}$ Raman and ATR-FTIR were also used for caliber differentiation but they applied low-level data fusion, in which both spectra were fused in a row-wise manner as an augmented matrix, after separate pre-processing. The authors reported that PLS-DA models for Raman alone showed 
Sn and Sp equal to $98 \%$ and for ATR-FTIR, 94 and 97\%, respectively. The combined augmented data provided perfect classification, showing improvement over classification using both techniques alone, as reported previously. ${ }^{142,145}$

In the third study, Bueno and Lednev ${ }^{144}$ analyzed GSR particles collected from a cotton cloth using a double-sided adhesive tape. The particles had been from a $0.38 \mathrm{~mm}$ revolver at close range $(30 \mathrm{~cm})$. In this case, Raman imaging (laser excitation $785 \mathrm{~nm}$ ) was used to analyze a $2 \mathrm{~mm}^{2}$ area of the sample. The data, comprised of 785 spectra, was divided into training and prediction sets and samples were divided into four classes using PLS-DA: tape, OGSR, IGSR and the unassigned group. Due to experimental issues, the authors reported that a GSR particle needed to be larger than $3.4 \mu \mathrm{m}$ for correct identification. The authors reported two types of misclassification of the spectra collected at the borders of the GSR particles: OGSR particles which were classified as tape (false-negative) and tape which was classified as GSR (false-positive). The misclassification results were due to the size of the laser beam, that reads contributions of both GSR and tape. Although the authors stated that the false-positive cases were not technically wrong, since this only happened in the presence of GSR particles, 104 spectra from the borders were removed and the classification results of unknown samples were improved: $93.7 \%$ of correct classification were obtained for OGSR, $90.4 \%$ for IGSR and $98.9 \%$ for tape. No samples were classified as unassigned.

López-López et al. ${ }^{146}$ also analyzed GSR particles from 4 types of ammunition (three conventional of different caliber and one non-toxic) using Raman imaging (laser excitation $455 \mathrm{~nm}$ ). Samples were analyzed directly on clothing and particles collected with an adhesive carbon tape on a stub. Instead of using a classification technique, they applied MCR-ALS to discriminate between the GSR and the substrate. They also added blood to some samples to evaluate the discriminating potential of the method with a possible interference. The GSR particles from the conventional and non-toxic ammunition were clearly identified. In addition to chemical information, this approach provided microscopic visualization of the GSR particles.

\subsection{Infrared spectroscopy}

In the same manner as Raman, IR spectroscopy is advantageous for detection of OGSR from the propellant because the spectra are highly selective, reproducible and analysis is nondestructive. However, very few studies have reported the use of chemometrics for GSR analysis using IR spectroscopy.
Bueno et $a l .{ }^{145}$ analyzed GSR from three different ammunitions (0.38, 0.40 and $9 \mathrm{~mm}$ calibers) on a cloth substrate at close distance $(30 \mathrm{~cm})$ using ATR-FTIR. For PLS-DA, the training set consisted of 160 GSR spectra and the prediction set consisted of thirty GSR spectra from additional discharges. All unknown GSR particles were assigned to the correct class of caliber in the validation and 93.3\% GSR particles from new discharges were correctly classified in the prediction.

Ortega-Ojeda et al. ${ }^{147}$ were the first to employ NIR imaging and CLS to discriminate GSR patterns from conventional and non-toxic $9 \mathrm{~mm}$ caliber ammunitions on white, black, or white-stamped cotton fabrics. For CLS, they tested two calibration sets, one consisting of spectra of isolated propellants from both types of ammunitions and another composed of pure standards of common compounds found in ammunition. In the first calibration set, the relative CLS concentration maps showed that in the conventional ammunition, the GSR patterns seemed to be bigger than the patterns observed in the target shots with the non-toxic ammunition. Nevertheless, the authors expressed concerns about using this method since not all forensic laboratories have large enough sample libraries containing sufficient types of ammunition propellants to be used as standards. Using the second calibration set, the strongest signals found in all targets belonged to nitrocellulose; other components were also detected, such as diphenylamine, nitroguanidine, 4-nitro-ethyl-centralite, and 2,6-dinitrotoluene. The authors argued that this calibration set is more versatile as the standards of different ammunition components are commercially available. They recommended the use of nitrocellulose as standard. Thus, the lack of fit responses for conventional and nontoxic ammunition using the recommended calibration set for white and black cotton were $39.8 \%$ (conventional on white cotton), $39.3 \%$ (non-toxic on white cotton), $31.3 \%$ (conventional on black cotton) and $21.3 \%$ (non-toxic on black cotton). Values of $\mathrm{R}^{2}$ were approximately 0.84 for both types of ammunition on white-cotton fabric and higher than 0.90 for black cotton, while the correlation coefficients presented values around 0.94 .

Weber and co-workers ${ }^{148,149}$ proposed the incorporation of luminescent markers in ammunition, based on metalorganic frameworks (MOFs), to facilitate the detection and identification of GSR from non-toxic ammunition. Furthermore, they argued that adjusting the chemical composition of luminescent markers opens up new perspectives for ammunition encoding. In this context, Carvalho et al. ${ }^{150}$ proposed the use of NIR imaging and MCR-ALS to discriminate among GSR particles containing three different MOFs based markers ([Eu(DPA) 
(HDPA)], $[\mathrm{Eu}(\mathrm{BTC})]$ and $\left.\left[\mathrm{Eu}_{2}(\mathrm{BDC})_{3}\left(\mathrm{H}_{2} \mathrm{O}\right)_{2}\right]_{\mathrm{n}}\right)$. The luminescent GSR (LGSR) particles were collected from weapons, hands and cartridges using five different tapes (masking tape, double sided, Teflon tape, adhesive paste and conductive carbon tape) placed in a stub. Initially, a set of 45 samples was collected to verify the most suitable substrate for the NIR analysis. Then, 19 samples that had been collected using the masking tape were analyzed to develop the MCR-ALS models and 18 other samples were used as a blind test. Spectra of the four pure components (tape and the luminescent markers) were employed for initial estimates. Only constraints for concentration were used: non-negativity, closure and equality constraints. An augmented MCR-ALS (column-wise) was employed to minimize the risk of rank deficiency by inserting matrices related to the spectra of the four possible components present in the samples. Evaluation of the recovered spectra and the relative concentration maps detected and correctly identified $72.2 \%$ of the samples. The misclassifications were related to little amount of material collected (8.3\%) or confusion caused by one of the markers, [Eu(BTC)] (19.4\%). The authors recommend that the [Eu(BTC)] should not be used for selective tagging and ammunitionencoding but could be used for GSR detection and identification using the methodology described.

Table 2 summarizes the research works and chemometric techniques for GSR.

In short, confocal and HSI Raman were selected for most GSR analyses performed directly on clothing or adhesive substrates. The $785 \mathrm{~nm}$ laser excitation was preferred in this case. PCA was used first, then several classification techniques, such as GLSW, k-NN, PLS-DA and SVM-DA, were used for discrimination among ammunition types. The MCR-ALS resolution technique was preferred for HSI applications. ATR-FTIR and HSI-NIR were both used for GSR analysis directly on clothing or adhesive substrates. Then, different approaches were used for identification of ammunition, such as the PLS-DA classification technique, the CLS calibration technique, and the MCR-ALS resolution technique.

\section{Explosives}

Explosives are highly energetic, sensitive and dangerous substances, used in military and commercial applications as well as in terrorist attacks. The investigation of explosives can be very complex and challenging because there are a variety of explosive compounds (different physical states or composition), from military, commercial and improvised sources, and they can be found intact, hidden in containers or found as trace amounts as post-blast residues. For further reading, refer to Huri et al. ${ }^{151}$ and López-López and García-Ruiz ${ }^{152}$ works.

\subsection{Raman spectroscopy}

Stewart et al. ${ }^{153}$ proposed a semi-quantitative method for determining the concentration of hydrogen peroxide using a portable Raman (laser excitation $785 \mathrm{~nm}$ ). First, they prepared a calibration curve from the peak height of five hydrogen peroxide concentrations (5, 10, 15, 20 and 25\%). They normalized the spectra to the band of the internal standard, sodium perchlorate, and obtained an average error of $1.43 \%$. They also did a PLS regression and obtained an average error of $0.98 \%$. Although the authors stated that the difference was not significant and that the univariate method is simpler, no significance test was mentioned for model comparison. Finally, they introduced their data into

Table 2. Resume of techniques for gunshot residues (GSR)

\begin{tabular}{|c|c|c|c|}
\hline Analytical technique & Chemometric technique & Sample & Reference \\
\hline Confocal Raman (785 nm) & PCA $,{ }^{a} k-N N,{ }^{b}$ PLS-DA, ${ }^{b}$ SVM-DA ${ }^{b}$ & GSR on clothing ( 2 ammunition types) & 142 \\
\hline Raman, ATR-FTIR & PCA, ${ }^{a}$ PLS-DA ${ }^{b}$ & GSR on clothing ( 2 ammunition types) & 143 \\
\hline HSI-Raman (785 nm) & PLS-DA ${ }^{\mathrm{b}}$ & $\begin{array}{l}\text { OGSR and IGSR on double-sided adhesive tape } \\
\text { ( } 2 \text { ammunition types })\end{array}$ & 144 \\
\hline HSI-Raman (455 nm) & MCR-ALS ${ }^{c}$ & $\begin{array}{c}\text { GSR on adhesive carbon tape and stub } \\
\text { (4 ammunition types })\end{array}$ & 146 \\
\hline ATR-FTIR & PLS-DA ${ }^{\mathrm{b}}$ & GSR on clothing ( 3 ammunition types) & 145 \\
\hline HSI-NIR & $\mathrm{CLS}^{\mathrm{d}}$ & $\begin{array}{l}\text { GSR on white, black and white stamped cotton fabric } \\
\qquad(2 \text { ammunition types })\end{array}$ & 147 \\
\hline HSI-NIR & MCR-ALS ${ }^{c}$ & GSR (luminescent markers) on 5 adhesive tapes & 150 \\
\hline
\end{tabular}

${ }^{\mathrm{a}}$ Exploratory; ${ }^{\mathrm{b}}$ classification; ${ }^{\mathrm{r}}$ resolution; ${ }^{\mathrm{d}}$ calibration. PCA: principal component analysis; k-NN: k-nearest neighbors; PLS-DA: partial least squaresdiscriminant analysis; SVM-DA: support vector machines-discriminant analysis; ATR-FTIR: attenuated total reflectance-Fourier transform infrared spectroscopy; HSI: hyperspectral images; OGSR: organic gunshot residues; IGSR: inorganic gunshot residues; MCR-ALS: multivariate curve resolutionalternating least squares; NIR: near infrared; CLS: classical least squares. 
the instruments library and compared the matching results with a series of testing solutions with different hydrogen peroxide concentrations $(5,7,15,17,25$ and $27 \%)$, for field applications. The library gave an average error of $1.16 \%$, which was comparable to the other methods. However, the potential sources of error in real case scenarios may arise from the evidence having been contaminated by perchlorate, which would lead to the underestimation of hydrogen peroxide.

López-López et al. ${ }^{154}$ analyzed thirty-three solutions of different types of nitrocellulose based gunpowder (single-base with and without dinitrotoluene, double-base containing nitroglycerin and triple-base) by FTIR and confocal Raman spectroscopy (laser excitation $532 \mathrm{~nm}$ ). Visual comparison of the FTIR and Raman spectra allowed differentiation only with the gun powders containing dinitrotoluene and with the triple-base gunpowders. Raman determined the presence of other minor components in smokeless gunpowder, such as diphenylamine and centralite. After band assignation, LDA using the Mahalanobis distance was used for classification. Six classes were created considering the type of gunpowder (single-base or double-base) and the presence or absence of components such as dinitrotoluene, diphenylamine or dibutyl phthalate. Raman spectroscopy showed better performance in discriminating gunpowders with or without diphenylamine and dinitrotoluene, and FTIR performed better in differentiating single-base gunpowder with or without dinitrotoluene, as well as double-base gunpowder.

Choo and co-workers ${ }^{155}$ applied PCA and a maximum a posteriori (MAP)-LDA method for classification of 14 explosive powders of different types (nitroaromatics, nitramines, nitrate esters, and peroxides). MAP is a discriminative Bayesian estimation method that models the probability density of each class using parameters from the training set, such as mean and covariance. Samples are assigned to the "most likely" class based on a feature vector with the highest posterior probability. ${ }^{156}$ They analyzed the samples using a benchtop (laser excitation 514.5 and $633 \mathrm{~nm}$ ) and a portable Raman instrument (laser excitation $785 \mathrm{~nm}$ ). The PCA results showed clear differences between the explosives; but there was a wide dispersion among spectra of the same type of explosive. Due to this within-group dispersion and the small number of training samples, only 10/14 explosives were correctly classified. Nevertheless, the authors reported an overall classification rate of $99.3 \%$.

Poppi and co-workers ${ }^{157,158}$ published two studies for the identification of explosive traces in banknotes using the same Raman imaging instrument (laser excitation $785 \mathrm{~nm}$ ). In the first, ${ }^{157}$ they developed the methodology for the identification of pre-blast traces of eight explosives (three military, four commercial and one propellant) on banknotes. They also analyzed different concentrations of trinitrotoluene (TNT), for investigating detection limits. The methodology consisted of applying MF-ICA to recover the spectra and identify the distribution maps for each component of the explosives. They used EFA for selecting the number of components necessary, because this information is unknown in real case scenarios and this number varied for each explosive. ICA showed correlation coefficients between the spectrum of reference and the profiles recovered close to 1 for all but three explosives. These three explosives, nonetheless, were unequivocally identified from their Raman bands. The method also made it possible to detect a level of TNT as low as $50 \mu \mathrm{g} \mathrm{cm}^{-2}$. The MCR-ALS model's lack of fit (LOF) and correlation coefficients were comparable to ICA's, with exception of TNT, where the MCR-ALS was not able to obtain satisfactory correlations between the recovered and the reference spectra. The authors justified this because of the absence of pure pixels and the low signal of the explosive TNT in the global model. The same method was then applied to the identification of explosives using real banknotes. MF-ICA found traces of carbon, sulfur and potassium nitrate in the Raman images of the banknote, which confirmed the presence of post-blast residues from black powder explosive.

In the second study, Poppi and co-workers ${ }^{158}$ applied the same methodology to the identification of post-blast explosive traces from an ATM explosion simulation experiment. Banknotes were contaminated with ANFO (ammonium nitrate fuel oil) and rhodamine B, which is a pigment that is usually added to the ATM as a safety device to render the banknotes unusable after explosions. The models used up to eight components; thus, the recovered spectra of each compound were not completely pure, due to the complexity of the matrix. Also, the LOF and explained variance were high (15.80 and $92.09 \%$, respectively). Nonetheless, based on the specific band of $\mathrm{NO}_{3}^{-}$symmetric stretching at $1044 \mathrm{~cm}^{-1}$, it was possible to identify the presence of ANFO and its location in the sample.

\subsection{Infrared spectroscopy}

Banas et al. ${ }^{159}$ highlighted the importance of using multivariate analysis instead of FTIR spectral libraries of explosives. They analyzed post-blast debris from three high-explosive compounds: C-4, TNT and pentaerythritol tetranitrate (PETN). HCA and PCA were used to visualize clustering of samples but PCA showed better performance. PCA was also employed by the study of Chen et al., ${ }^{160}$ 
in which fingerprints contaminated with a hexahydro1,3,5-trinitro-1,3,5-triazine (RDX) explosive solution were analyzed by FTIR imaging. The PCA scores images and the loadings enabled differentiation of the distribution of the retrieved eccrine/sebaceous components of gland secretions and the distribution of RDX.

Regarding classification applications, de la Ossa et al. ${ }^{161,162}$ published two papers dealing with the use of NIR imaging for the detection of residues of different explosives on hands. The advantage of the NIR system, in comparison with the MIR system employed in the study reported in, ${ }^{160}$ is that it allows the scanning of the entire handprint, increasing the possibility of detecting the presence of explosive residues. Hands of different volunteers which had been contaminated with different kinds of explosives (ammonium nitrate, black powder, single- and double-base smokeless gunpowder and dynamite) were pressed on plastic sheets, similar to that used for handprint collection. The calibration set for the PLS-DA models consisted of a 50-pixel windows obtained from the individual images of each explosive. The crossvalidation confusion matrix showed that all nitrocellulose, ammonium nitrate, dynamite, black powder and plastic classes were correctly classified in their respective class but lower classifications were observed for single- and double-base smokeless gunpowder, possibly because they are mainly composed of nitrocellulose and showed similar NIR signals. Nevertheless, they were differentiated from the remainder classes. The authors remark that further studies are important to estimate the limits of detection, the mass transfer rates from explosives to hands and to sheets, and possible interferences.

A direct and fast procedure of sampling and analysis of consumer firework post-blast residues using a portable ATR-FTIR instrument was proposed by MartínAlberca et al. ${ }^{163}$ The spectra of the post-blast residues from five common consumer fireworks were obtained directly from the three different cotton swabs tested using the ATR crystal. 22 consumer fireworks were also analyzed to trace back the signal of the post-blast residues to the original pyrotechnic compositions. PCA showed different results depending on the swab used and the collecting place. The authors recommended more research to find an appropriate collection device for sampling a wider variety of substrates.

A portable NIR was used with PCA and PLS-DA models for the detection of explosives on human hands by Risoluti et al. ${ }^{164}$ Three experiments were conducted to investigate the application to real cases, such as the ability to detect explosives in complex matrices, the effects of interferences from other explosive materials that evaluates the ability of the model to detect more than one explosive at the same time, and the persistence of the residuals over time after daily hand washing. From the twenty-five samples from the volunteers involved, 10 were included in the training set, 5 to model validation and the other 10 to prediction. PLS-DA models were also built using 5 standard materials (TNT, PETN, RDX and the composites DEMEX and M75) to evaluate the matrix effects on the identification of the explosives. PCA was performed using the spectra obtained from 15 volunteers to whose hands had been contaminated with increasing amounts of explosives, to simulate the handling of those materials. Distinct clusters from each one of the compounds were observed. PLS-DA validation provided classification rates of $80 \%$ for M75, 91.7\% for DEMEX, $96.7 \%$ for TNT and $100 \%$ for PETN and RDX. Results from prediction showed $100 \%$ correct classifications rates of all materials. Moreover, the ability to detect the residuals using the method proposed decreased as the time from handling increased, which is comparable with the results obtained with the reference method gas chromatography-mass spectrometry (GC-MS), even after a period of $24 \mathrm{~h}$. Among the investigated explosives, both DEMEX and M75 proved to be the most persistent on the hands, even after washing, whereas TNT had the worst resistance after daily activities.

Table 3 shows the main reference mentioned for explosives.

As shown, both confocal, HSI and portable Raman were used for identification of several explosives, particularly using the $785 \mathrm{~nm}$ laser excitation. The exploratory techniques PCA and MF-ICA were applied for preliminary analysis and then, either the classification techniques PLS and LDA or the resolution technique MCR-ALS were used for HSI analysis of explosives on banknotes. FTIR was used for pre- and post-blast explosive analysis, while HSI-NIR was preferred for hand- and fingerprint analysis of explosive traces. PCA was preferred in most studies and PLS-DA for subsequent analysis of handprints.

\section{Illicit Drugs}

Investigations of illicit drugs comprise the largest load of work by forensic science laboratories, whether it is for the identification of unknown substances, the quantification of the active compound(s) and major adulterants, the profiling for intelligence purposes of bulk seizures or for biological and non-biological matrices. Besides interfering matrices, the complexity of drug investigations resides in the fact that the materials under study contain a variety of substances and often require laborious and time-consuming chemical separations. 
Table 3. Resume of techniques for explosives

\begin{tabular}{|c|c|c|c|}
\hline Analytical technique & Chemometric technique & Sample & Reference \\
\hline Portable Raman (785 nm) & $\mathrm{PLSR}^{\mathrm{a}}$ & concentration of hydrogen peroxide & 153 \\
\hline Confocal Raman (532 nm), FTIR & $\mathrm{LDA}^{\mathrm{b}}$ & solutions of nitrocellulose based gunpowders & 154 \\
\hline $\begin{array}{l}\text { Confocal Raman }(514,633 \mathrm{~nm}) \text {, } \\
\text { portable Raman }(785 \mathrm{~nm})\end{array}$ & $\mathrm{PCA},{ }^{\mathrm{c}} \mathrm{LDA}^{\mathrm{b}}$ & 14 explosive powders & 155 \\
\hline HSI-Raman (785 nm) & MF-ICA, ${ }^{\mathrm{c}}$ MCR-ALS ${ }^{\mathrm{d}}$ & pre-blast traces of 8 explosives in banknotes & 157 \\
\hline HSI-Raman (785 nm) & MF-ICA, ${ }^{c}$ MCR-ALS ${ }^{d}$ & post-blast traces of ANFO in banknotes & 158 \\
\hline FTIR & $\mathrm{PCA},{ }^{\mathrm{c}} \mathrm{HCA}^{\mathrm{c}}$ & post-blast traces of 3 high explosives & 159 \\
\hline HSI-FTIR & $\mathrm{PCA}^{\mathrm{c}}$ & solution of RDX in fingerprints & 160 \\
\hline HSI-NIR & $\mathrm{PCA}^{\mathrm{c}}{ }^{\mathrm{PLS}}-\mathrm{DA}^{\mathrm{b}}$ & pre-blast on hand & 161,162 \\
\hline FTIR & $\mathrm{PCA}^{\mathrm{c}}$ & pre-blast and post-blast fireworks on cotton swabs & 163 \\
\hline Portable NIR & PCA, ${ }^{\mathrm{c}}$ PLS-DA ${ }^{\mathrm{b}}$ & 4 explosives on plastic handprints & 164 \\
\hline
\end{tabular}

${ }^{\mathrm{a} C a l i b r a t i o n ;}{ }^{\mathrm{b}}$ classification; ${ }^{\mathrm{c} e x p l o r a t o r y}$; ${ }^{\mathrm{r}}$ resolution. PLSR: partial least squares regression; FTIR: Fourier transform infrared spectroscopy; LDA: linear discriminant analysis; PCA: principal component analysis; HSI: hyperspectral images; MF-ICA: mean field independent component analysis; MCR-ALS: multivariate curve resolution-alternating least squares; HCA: hierarchical cluster analysis; NIR: near infrared; PLS-DA: partial least squares-discriminant analysis.

\subsection{Raman spectroscopy}

All studies recently published on identification of illicit drugs by Raman spectroscopy and chemometrics have focused in SERS analysis of drugs in body fluids, like urine and saliva, due to the need for sensitive detection in complex matrices. Liu and co-workers ${ }^{165,166}$ published two studies for the identification and quantification of amphetamines in human urine based in SERS analysis, where in the first, ${ }^{165}$ they analyzed samples of urine mixed with different concentrations of the drug $(0,0.01,0.1,1$, 10 and $100 \mathrm{ppm}$ ) using a portable Raman instrument (laser excitation $785 \mathrm{~nm}$ ). For SERS, gold nanorod substrates were used and a different and simplified analysis procedure was performed, where the sample was analyzed as it dried on a surface, rather than waiting for complete drying, as in a conventional SERS method. SVM was applied to classify different ratio proportions of two methamphetamines in human urine. Calibration models were built using the SERS spectra of urine of 50 humans with and without drugs and in different concentrations (50, 2.5 and $0.1 \mathrm{ppm})$. They obtained a classification accuracy of $96 \%$ for one of the drugs; the accuracy for the lowest concentration (0.1 ppm) was $94 \%$, as expected. This result was $11 \%$ higher than with the conventional SERS procedure. The model validation with urine samples from three real drug abusers showed classification accuracy higher than $90 \%$. The authors defended the method as rapid ( 2 min analysis time) and practical ( $2 \mu \mathrm{L}$ sample volume) for on-site application. In the second study, ${ }^{166}$ they used an oil-in-water emulsion made from silver nanoparticles and a surfactant as SERS substrates. Drugs were first extracted from human urine with cyclohexane in strong alkaline media. SERS analysis enabled a limit of detection of $10 \mathrm{ppb}$. Besides ultratrace identification, PCA (for data reduction) and SVM-DA classification resulted in $98 \%$ accuracy for different proportions of the two methamphetamines in human urine.

Mabbott et al. ${ }^{167}$ deposited silver nanoparticles on British coins to create substrates for SERS analysis of mephedrone and two amphetamines (5,6-methylenedioxy-2-aminoindane (MDAI) and 3,4-methylenedioxymethamphetamine (MDMA)). PCA was used to assess the reproducibility of the SERS method and PLS-DA was then used for classification. They created three separate models for each drug and used bootstrap validation (1000 iterations). Overall, the specificity, precision and accuracy for the three drugs was higher than $95 \%$. The models displayed an excellent sensitivity for MDAI and mephedrone but lower for MDMA due to the high number of false negatives identified. The authors mentioned that the loadings showed discriminating vibrational modes for each drug.

Andreou et al. ${ }^{168}$ developed a microfluidic device for SERS trace detection of amphetamines in saliva, using PCA and Varimax rotation. After rotation, they were able to identify the different SERS bands in each LV that were related to the illicit drug. Based on this, they determined methamphetamine concentrations as low as $10 \mathrm{nM}$. Despite the results, the authors made an erroneous statement by saying that PCA is a tool for automated classification of spectra.

D'Elia et al. ${ }^{169}$ used gold nanorods for SERS identification and quantification of cocaine in oral fluids using a confocal Raman (laser excitation $785 \mathrm{~nm}$ ). OPLS-DA was used to discriminate among oral fluid solutions with various 
cocaine concentrations. Although authors did not give details on the construction of the models, it was clear that the data set was small. Despite this, the results showed clear differentiation between the samples; and the model was able to differentiate the $1 \mathrm{ng} \mathrm{mL}-1$ concentration of cocaine from the oral fluid. The authors stated that this method could be used for quantification by enlarging the number of drug concentrations, sample replication, and the inclusion of oral fluids from different donors.

Dies et al. ${ }^{170}$ used silver nanoparticle substrates which were electrochemically immobilized for SERS identification of different drugs (cocaine, heroin, tetrahydrocannabinol (THC) and oxycodone) in oral fluid using a confocal Raman (laser excitation $633 \mathrm{~nm}$ ). The SVM technique with leave-one-out cross validation was used for classification, although not many details on model building and sampling were provided. As a result, $100 \%$ classification accuracy was obtained for drugs in solution and the developed model was able to classify $98.3 \%$ of the different concentrations of cocaine samples.

Massarini et al..$^{171}$ used commercial SERS substrates made of gold inverted pyramids for SERS analysis of solutions of ten narcotic drugs (amphetamine, cocaine, methadone, diazepam, methylphenidate, oxazepam, tramadol, morphine, buprenorphine and 6-monoacetylmorphine) in different concentrations. Confocal and a portable Raman instruments (laser excitation $785 \mathrm{~nm}$ ) were used. Limits of detection were obtained directly from spectra and limits of identification were obtained using PLS-DA models. Because it was not possible to obtain at least three spectra for the 6-monoacetylmorphine, the buprenorphine and the morphine with sufficient signal-to-noise ratio, they were excluded from the model. The test set consisted of 42 spectra from the seven drugs and the validation set consisted of 149 spectra from the same seven drugs at different concentrations in a solvent, which were not used in the test set. Results showed that only one amphetamine sample was misclassified as cocaine. When comparing the PLS-DA results with the conventional method for calculating the limit of identification (two times the limit of detection), the chemometric method performed better, with exception of methylphenidate, possibly because these observations were made under sparse concentrations.

\subsection{Infrared spectroscopy}

The studies published on illicit drugs by IR spectroscopy and chemometrics focused in the analysis of confiscated bulk drugs. An important issue to consider in the analysis of confiscated drugs is the representativeness of calibration and prediction sets, due to the large complexity and variability of the samples. This was already remarked in 1999 by Sondermann and Kovar, ${ }^{172,173}$ one of the first studies on the matter. Since then, several studies have reported the use of benchtop and portable NIR and MIR instruments with multivariate calibration models (mostly PLS), taking into consideration calibration sets that are representative for the quantitative analysis of illicit drugs in tablets/powder, such as methylamphetamine, ${ }^{174}$ heroin ${ }^{175}$ and cocaine. ${ }^{176-180}$

Pérez-Alfonso et al. ${ }^{181}$ used PLS regression to determine the content of cocaine impregnated in seized materials, within which were found other forms of illicit drugs. Measurements were made by ATR-FTIR and diffuse reflectance (DR) NIR directly from the surface of four different types of materials, white and black textiles, paper and foam. These materials were impregnated with cocaine concentrations $(\mathrm{m} / \mathrm{m})$ between 38.1 and $54.1 \%$ for textiles, $50.7 \%$ for paper and $68.1 \%$ for foam. PLS models were built using the textile samples. PCA was used to evaluate the similarity of the calibration set with new samples. The authors concluded that PLS was adequate (RMSECV and RMSEP of approximately 4\%) when the drug was distributed homogenously, even though the matrix could be different.

Two studies ${ }^{182,183}$ employed supervised pattern recognition methods and IR to classify cocaine samples according to their form (base or salt), content, and adulterants. Rodrigues et al. ${ }^{182}$ used ATF-FTIR to analyze 91 samples of apprehended cocaine powder. PCA results showed the presence of lidocaine, caffeine and benzocaine as well as information related to the chemical form of cocaine. Two PLS-DA models were built to discriminate among the concentrated, diluted, salt and base cocaine samples. They showed rates of true positives between 95 and $97 \%$, and of true negatives between 83 and $88 \%$. Marcelo et al. ${ }^{183}$ analyzed 500 samples and used HCA and PCA to evaluate patterns in the data and PLS-DA and SVM to classify the samples according to their form. The training and prediction sets were composed by 100 and 413 samples, respectively. They obtained $100 \%$ sensitivity and specificity with both methods.

Liu et al. ${ }^{184}$ proposed a two-step qualitative and quantitative classification approach to determine the content of methamphetamine, ketamine, heroin, and cocaine in confiscated samples, using NIR spectroscopy and classification methods. A total of 282 samples were used for calibration and 836 samples were used for prediction. The classification step was used to decide what class the sample belonged to (methamphetamine, ketamine, heroin, and cocaine or none of these). With this information, the sample was directed to the corresponding PLS model. Then, SIMCA and SVM were evaluated with the first chosen due 
to better performance for rejecting false positive results. The methodology was considered accurate for qualitative and quantitative analysis of drug samples. A similar approach was employed by Deconinck et al. ${ }^{185}$ for 267 seized ecstasy tablets and other party drugs, also using DR-NIR and ATR-FTIR. PLS-DA was used for classification and PLS calibration for quantification of MDMA tablets. In the qualitative classification, the DR-NIR gave the best results with $96 \%$ correct classification for the prediction set. The errors obtained, however, were false-positives. Given this, the authors warned against routine applications because the follow up analysis would include PLS quantification and would result in wrong conclusions. Therefore, falsenegatives could be less problematic since samples would be sent to a laboratory for confirmatory analysis. The authors suggested that the only way to tackle this problem is to update the model regularly with new samples, rendering the model more robust and more reliable.

Some studies have reported on the analysis of new psychoactive substances (NPS), which are a large group of synthetic drugs frequently found in the drug market. Risoluti et al. ${ }^{186}$ analyzed synthetic cannabinoids and phenethylamines using DR-NIR. Standards of these substances alone and in simulated matrices (dried herbs and blotter papers) were also analyzed to determine the matrix influence on the spectroscopic signal. PCA was initially used to differentiate among the type of drugs. The scores of the simulated and confiscated samples showed some clustering according to the substances investigated even when dispersed in the matrix tested.

Pereira et al. ${ }^{187,188}$ published two studies related to this. In the first, ${ }^{187}$ they analyzed 73 seized samples of blotters containing NPS and 21 similar papers without drugs using ATR-FTIR and a hierarchical approach of sequential modeling using PLS-DA. The models were relatively successful in classifying samples into three classes (NBOMe, 2C-H and methallylescaline) and in differentiating the drug from the blank papers. The average of ACC was $91.1 \%$, CON was $86.1 \%$ and reliability rate (RLR) was $88.9 \%$. The RLR express a global rate of true positives and negatives. ${ }^{189}$ The authors also tried to discriminate among lysergic acid diethylamide (LSD) samples but without success because of the low concentrations present and the limited sensitivity of the technique. A sub model was then built to classify different NBOMe samples. RLR, ACC and CON results were 82.2, $100,94.4 \%$, respectively. After careful spectral interpretation using the VIP scores, the authors mentioned that the small number of samples in each class did not allow for more reliable models but the potential of the methodology had been demonstrated. In the second study, ${ }^{188}$ they used the same method to classify confiscated ecstasy-like tablets containing cathinone, tryptamines and other amphetamine types, including the well-known MDMA. The PLS-DA model discriminated four classes of substances: 5-MeOMiPT, MDMA, 3,4-methylenedioxy-amphetamine (MDA), methamphetamine and cathinone. The RLR and ACC was 96.8 and $100 \%$, respectively. Then, two sub-models were built to identify drugs present in the MDMA, MDA and cathinone classes. A total of 92 tablet samples were used to build the models and for validation. Since the number of samples for each class was very limited, only crossvalidation was employed. The RLR and ACC were $100 \%$.

Table 4 is a resume of the above-mentioned papers and their respective chemometric techniques for illicit drugs.

Regarding Raman, SERS was the technique of choice for drug analysis in biologic matrices (urine and saliva) using the 633 and $785 \mathrm{~nm}$ laser excitations. PCA was combined with several discriminant analysis techniques like SVM-DA, PLS-DA and OPLS-DA. ATR-FTIR was applied for direct and bulk analysis of illicit drugs. PCA was used preliminary and then, discriminant analyses like PLS-DA and SVM-DA were applied. The calibration technique PLSR was used to determine the concentration of active compounds in drug apprehensions.

\section{Body Fluids}

Detection and identification of body fluids at a crime scene is perhaps one of the most important findings in a criminal investigation because it can provide a DNA profile that links a suspect to the crime and its presence can help determining what happened. The difficulty with most investigations is that body fluids in very small amounts can be invisible to the naked eye, they can be heterogeneous or mixed with other substances and found on different types of surfaces. Consequently, examinations should be sensitive, selective and non-destructive, particularly concerning DNA. The most relevant body fluids include blood, semen, saliva and urine.

\subsection{Raman spectroscopy}

Raman spectroscopy is a useful technique for the analysis of biological materials because the spectra are informative and present characteristic bands that can be linked to specific body fluids. The recognition of this analytical tool for body fluids has been due to the extensive work of Lednev and co-workers. ${ }^{190-198}$ They published a series of similar studies over four years using a similar methodology for the analysis of different body fluids (semen, ${ }^{190}$ saliva, ${ }^{191}$ blood, ${ }^{192}$ sweat $^{193}$ and vaginal 
Table 4. Resume of techniques for illicit drugs

\begin{tabular}{|c|c|c|c|}
\hline Analytical technique & Chemometric technique & Sample & Reference \\
\hline SERS (785 nm) (portable) & SVM-DA $^{\mathrm{a}}$ & amphetamines in human urine & 165 \\
\hline SERS & $\mathrm{PCA}^{\mathrm{b}}{ }^{\mathrm{b}} \mathrm{SVM}-\mathrm{DA}^{\mathrm{a}}$ & methamphetamine in human urine & 166 \\
\hline HSI-SERS (633 nm) & PCA, ${ }^{\mathrm{b}}$ PLS-DA ${ }^{\mathrm{a}}$ & mephedrone and two amphetamines & 167 \\
\hline SERS & $\mathrm{PCA}^{\mathrm{b}}{ }^{\text {Varimax }}$ rotation $^{\mathrm{b}}$ & amphetamines in saliva & 168 \\
\hline SERS (785 nm) & OPLS-DA $^{\mathrm{a}}$ & cocaine in saliva & 169 \\
\hline SERS $(633 \mathrm{~nm})$ & SVM-DA $^{\mathrm{a}}$ & cocaine, heroin, THC, oxycodone in saliva & 170 \\
\hline SERS (785 nm) & PLS-DA ${ }^{a}$ & 10 narcotic drugs in different concentration & 171 \\
\hline ATR-FTIR & $\mathrm{PCA}^{\mathrm{b}} \mathrm{PCR}^{\mathrm{c}}$ & mixtures containing methylamphetamine & 174 \\
\hline DR-NIR & $\mathrm{HCA}^{\mathrm{b}}{ }^{\mathrm{PLSR}}{ }^{\mathrm{c}}$ & heroin in street drugs & 175 \\
\hline NIR & $\mathrm{PLSR}^{\mathrm{c}}$ & cocaine & 176 \\
\hline ATR-FTIR & PLS-DA, ${ }^{\mathrm{a}}$ PLSR $^{\mathrm{c}}$ & cocaine and adulterants quantification & 177 \\
\hline ATR-FTIR & $\mathrm{PLSR}^{, c} \mathrm{PCR},{ }^{\mathrm{c}} \mathrm{MLR}^{\mathrm{c}}$ & cocaine and adulterants quantification & 178 \\
\hline Portable NIR & $\mathrm{PCA}^{,}{ }^{\mathrm{b}} \mathrm{HCA}^{\mathrm{b}}{ }^{\mathrm{PLSR}}{ }^{\mathrm{c}}$ & cocaine quantification, ecstasy, designer drugs, medicines & 179 \\
\hline ATR-FTIR & $\mathrm{PLSR}^{\mathrm{c}}$ & cocaine quantification & 180 \\
\hline ATR-FTIR, DR-NIR & PCA, ${ }^{\mathrm{b}} \mathrm{PLSR}^{\mathrm{c}}$ & $\begin{array}{c}\text { different concentrations of cocaine on white and black } \\
\text { textile, paper and foam }\end{array}$ & 181 \\
\hline ATR-FTIR & PCA, ${ }^{b}$ PLS-DA ${ }^{a}$ & 91 cocaine powder seizures and adulterants & 182 \\
\hline ATR-FTIR & PCA, ${ }^{b}$ HCA, ${ }^{b}$ PLS-DA, ${ }^{a}$ SVM-DA ${ }^{a}$ & 500 cocaine seizures & 183 \\
\hline NIR & PLSR, ${ }^{\mathrm{c}}$ SIMCA, ${ }^{\mathrm{a}}$ SVM-DA ${ }^{\mathrm{a}}$ & $\begin{array}{c}282 \text { methamphetamine, ketamine, heroin and cocaine } \\
\text { seizures }\end{array}$ & 184 \\
\hline DR-NIR, ATR-FTIR & PLS-DA, ${ }^{\mathrm{a}}$ PLSR $^{\mathrm{c}}$ & 267 ecstasy tablets and party drugs & 185 \\
\hline DR-NIR & $\mathrm{PCA}^{\mathrm{b}}$ & $\begin{array}{l}\text { synthetic cannabinoids and phenethylamines bulk and with } \\
\text { dried herbs and on blotter papers }\end{array}$ & 186 \\
\hline ATR-FTIR & PLS-DA ${ }^{\mathrm{a}}$ & $73 \mathrm{NBOMe}, 2 \mathrm{C}-\mathrm{H}$, methallylescaline on blotter papers & 187 \\
\hline ATR-FTIR & PLS-DA ${ }^{a}$ & cathinones, tryptamines and amphetamine tablets & 188 \\
\hline
\end{tabular}

${ }^{a}$ Classification; ${ }^{b}$ exploratory; ${ }^{c}$ calibration. SERS: surface-enhanced Raman spectroscopy; SVM-DA: support vector machines-discriminant analysis; PCA: principal component analysis); HSI: hyperspectral images; PLS-DA: partial least squares-discriminant analysis; OPLS-DA: orthogonal partial least squares-discriminant analysis; THC: tetrahydrocannabinol; ATR-FTIR: attenuated total reflectance-Fourier transform infrared spectroscopy; PCR: principal component regression; DR-NIR: diffuse reflectance-near infrared; HCA: hierarchical cluster analysis; PLSR: partial least squares regression; MLR: multiple linear regression; SIMCA: soft independent modeling of class analogy.

fluid) ${ }^{194}$ using the same confocal Raman (laser excitation $785 \mathrm{~nm}$ ). They evaluated the heterogeneity of fluids from the same donor and the variability between donors using curve fitting methods based on an ALS algorithm. Finally, spectral signatures of the different body fluids showed many fluorescence contributions, heterogeneity within the same donor and significant variations from donor to donor. Nevertheless, Raman enabled the identification of each body fluid. The same authors published another study ${ }^{195}$ for the discrimination of menstrual and peripheral blood from 20 and 21 donors, respectively. The Raman spectral profiles of both types of blood was very similar but PCA showed clear differences. However, the loadings were not shown nor discussed. Regarding classification, SVM-DA showed better sensitivity and specificity based on single spectrum (97.5 and 98\%, respectively), evidencing the nonlinearity of the dataset. For classification at the donor level, both models showed similar sensitivity (95\%) but SVM-DA gave $100 \%$ specificity. Another approach was proposed for improving the results of the previous models, consisting of excluding misclassified samples from the training set until all samples were correctly classified. This methodology must be carefully considered because of the high risk of overfitting. Instead, increasing the confidence level may ensure a high percentage of true positives. Finally, they performed SVM-DA using another class for vaginal fluid. As a result, no vaginal fluid samples were classified as menstrual or peripheral blood.

Two more studies ${ }^{196,197}$ were published using different classification techniques for the analysis of several body fluids. In the first, ${ }^{196}$ they used SIMCA, LDA and PLS-DA for discriminating among semen, blood and saliva from multiple donors. For the differentiation of these species, they applied PCA for data reduction and SIMCA. They used 
17 blood and saliva samples and 50 semen samples. The spectra were average for each sample and $100 \%$ of correct classification was obtained for all classes. Similarly, 100\% correct classification was obtained with LDA using either the leave-one-out cross-validation, naive Bayes classifiers, fitting of multivariate normal densities with covariance estimates stratified by group or Mahalanobis distances. Regarding PLS-DA, all species were clustered separately, with except for one Raman spectrum of human saliva that fell close to the human blood cluster. In the second study, ${ }^{197}$ they compared the use of SVM-DA and PLS-DA with and without GA and interval partial least squares (iPLS)-DA variable selection for discriminating peripheral blood, saliva, semen, sweat and vaginal fluid. Initial comparison between PLS-DA and SVM-DA models with the same dataset and using five latent variables showed more accurate predictions (internal and external validations) for SVM-DA, over $99 \%$ against $97 \%$ for PLS-DA. When using iPLS-DA variable selection, accuracy for the internal validation improved for both PLS-DA and SVM-DA but was lower than before for external validation. On the contrary, the accuracy of both models improved when using less than half of the variables. In fact, external validation with SVM-DA obtained $100 \%$ correct classification. The authors reported that GA analysis retained the distinctive peaks of each fluid, which is the reason for the satisfactory performance.

Mixtures of semen and blood in different ratios (varying from pure semen to pure blood) were analyzed in another study by the same authors. ${ }^{198}$ The first approach consisted of SVM-DA using only mixtures (total of 15 classes). Due to the disappointing results, the authors used another approach where SVM regression was applied before SVM-DA. Since the Raman spectra of mixtures outside of the $15-75 \%$ range could not be distinguished from the pure fluids, they were omitted from the training set. This allowed for $100 \%$ correct classifications. However, when including the omitted samples, most of the mixtures were correctly classified anyway in the cross-validation, with exception of the sample with 5\% blood that was assigned to pure semen. They obtained similar results in a validation study using mixture samples from six donors.

Lednev and co-workers ${ }^{199,200}$ have also published studies for differentiating human and animal blood. In one study, ${ }^{199}$ they used PCA for visualizing the differences between human, canine, and feline blood. The scores plot using 3 PCs showed three separate groups for each blood type, with more than $90 \%$ explained variance. In another study, ${ }^{200}$ they used PLS-DA for classification of human and animal blood from 11 species (cow, cat, dog, horse, pig, mouse, opossum, raccoon, rabbit, rat and chicken). The model was restrained to a binary classification of samples as either human or animal. Calibration was performed using 110 spectra from all the species, excluding ten cow samples. The cross-validation prediction using $4 \mathrm{LV}$ s showed that all samples were correctly classified with exception of two spectra from different chicken samples. The authors suggest that the use of additional LVs could correct this issue, but this has to be done carefully to avoid overfitting. Then, they performed external validation using the cow samples and ten unknown samples chosen blindly ( 5 human and 5 animal) and obtained $100 \%$ accuracy, in spite of a few samples that were very close to the threshold. The same authors recently expanded their dataset to include blood samples from 6 other animal species, chimpanzee, deer, elk, ferret, fish, and macaque. ${ }^{201}$ They also included new human donors varying in age (11-40+ years old). Results showed that most of the new animal species' blood samples were correctly predicted as non-human, with exception of samples from one chimpanzee and one macaque, which were classified as human blood. The authors were not surprised with this result because the hemoglobin structure of chimps is similar to humans. Apart from this, they reported $100 \%$ sensitivity and $93 \%$ specificity in external validation.

Bai et al. ${ }^{202}$ also published a similar study but with fewer animal species and obtained the same results. Fujihara et al. ${ }^{203}$ also analyzed human and animal blood from 11 species using a portable Raman instrument (laser excitation $785 \mathrm{~nm}$ ). However, the authors investigated spectral differences in bloodstains that had been aged for different periods. PCA scores plot showed no distinction between human and animal spectra, even after 3 months aging.

Lednev and co-workers ${ }^{204-206}$ published three studies for determining gender and race in bloodstains ${ }^{204,205}$ and sex in saliva. ${ }^{206}$ For gender discrimination employing blood samples, PCA showed no characteristic structure and GA was combined with artificial neural networks (ANN) to provide specificity and sensitivity of 81 and $81 \%$ for cross-validation, respectively. The SVM-DA model showed worse performance, with $73 \%$ for specificity and $79 \%$ for sensitivity. For saliva samples, an SVM-DA model was built employing 48 donors for training and 12 for prediction. The authors reported that the threshold values of 0.50 and 0.67 provided similar results, and they chose a threshold of 0.67 and correctly classified 11 out of 12 donors. For race discrimination, they also used GA for variable selection associated to SVM-DA models to classify samples according Caucasian and African American classes from 20 donors. The area under the receiver operating characteristic (ROC) curve, was used for evaluation, providing a value of 0.83 based on each subject. The authors 
explained that not all spectra have noticeable contributions from discriminating biomarkers.

The classification of blood samples contaminated with sand, dust and soil were evaluated with PLS-DA. ${ }^{207}$ Four classes were built for the training set, consisting of pure blood, dust, sand, and soil. The contaminated samples of blood were then predicted in that model. Results showed that there were no misclassifications of pure samples and a considerable numbers of contaminated blood spectra were assigned to blood. The authors did not show values of classification errors, sensitivity, specificity nor any figures of merit for the model; instead, they showed the PLS-DA prediction plot, from which many misclassified samples could be depicted. A similar approach was used for classification of semen stains on different substrates, like pig skin, glass, white cotton, blue polyester and white fabric composed of $60 \%$ cotton and $40 \%$ polyester. ${ }^{208}$ Since only a partial separation of samples was possible due to strong spectral contributions, MCR-ALS was employed for recovering the semen spectra. The authors showed a good fitting between the optimized spectra and the pure spectra of semen, especially on cotton, but found some difficulties with blue polyester.

Two studies ${ }^{209,210}$ were published regarding the age determination of bloodstains. At first, they used PLSR for age predictions and 2D correlation for spectral comparison. Blood samples from two donors (one male and one female) were left to age under normal laboratory conditions and spectra were measured at different times $(1,3,5,8,24,48$, $72,96,120$, and $168 \mathrm{~h}$ ). The PLSR model, built with data collected from the male donor, provided an RMSECV and $\mathrm{R}^{2}$ values of $0.13 \mathrm{~h}$ and 0.97 , respectively. The performance of the PLSR model was externally validated with a dataset from spectra of the female donor. It is important to emphasize here that a study of this complexity should consider more representativeness in the sampling procedure. For further investigation on that matter, inclusion of more donors and replicates of bloodstains from the same donor is strongly recommended. In the second study, they analyzed samples naturally aged up to 2 years. An SVM-DA model, which had been developed in a previous study ${ }^{197}$ was employed for making predictions of the aged bloodstains. As a result, all samples up to one month were correctly classified, which is understandable since the model was trained for fresh blood samples (up to $48 \mathrm{~h}$ ). For calibration, they built and compared PLSR with PCR and both calibration models provided very similar prediction results. However, the PLSR presented lower RMSEP value (0.29 compared to 0.31 of PCR). Additionally, the PLSR showed some variations in the accuracy of predictions at the different time points, but this lessened over time, possibly due to spectral quality.
More recently, Doty and Lednev ${ }^{211}$ attempted to use SVM-DA to classify blood samples from newborns (less than 1 year), adolescents (11-13 years old) and adults (43-68 years old). Results showed sensitivity values of $0.95,1.00$, and 0.98 , and specificity values of 1.00 , 0.98 , and 0.99 , for external predictions of the newborn, adolescent, and adult age classes, respectively. Although most misclassifications occurred between adolescent and adult classes, none of these were misclassified in the newborn class, which indicates the potential of the method to discriminate newborn from older blood samples.

\subsection{Infrared spectroscopy}

IR spectroscopy is somewhat less applicable for the analysis of body fluids than Raman because of the water content in most of the evidences. In addition to this, the higher penetration of IR in the evidence captures interfering signals from the substrates. Notwithstanding, several works have been published, particularly on dry stains. Mistek and Lednev $^{212}$ used ATR-FTIR and PLS-DA to discriminate between human and animal (cat and dog) blood deposited on microscope slides. GA was used for variable selection. Classification predictions of unknown blood donors resulted in $100 \%$ accuracy.

Two studies ${ }^{213,214}$ have used portable NIR spectrometers and supervised pattern recognition methods for discrimination and confirmatory identification of bloodstains in different substrates. In the first, Pereira et al. ${ }^{213}$ analyzed dried human and animal bloodstains and several red-colored products that could be commonly perceived as blood, such as red lipstick, pepper sauce, soy sauce, red wine and balsamic vinegar. The different substrates used included beige floor porcelain tile, white ceramic tile, glass and the metallic part of a knife. SIMCA, GA-LDA and SPA-PLSDA models were built for each substrate separately. For an external validation set, SIMCA showed $100 \%$ correct classification for porcelain and glass substrates but $80 \%$ for metal and $90 \%$ for ceramic substrates. GA-LDA showed better classification performance $(100 \%)$ than SPA-LDA, which gave one false positive and one false negative. PLSDA correctly classified human blood and other stains in all substrates. Morillas et al. ${ }^{214}$ analyzed bloodstains and eight other substances that resembled fresh or dry blood, such as red wine, red ink, tomato sauce, fake blood, coffee, red food coloring, red paint and beet root juice. The substrates used were glass slides, tile, wood, $100 \%$ leather, $100 \%$ acrylic and $100 \%$ cotton. The authors did not describe the chemometrics used because the models were created using the software tools provided by the manufacturer of the device, but they mentioned that these tools utilize a 
"powerful cloud-based machine learning" method which is based on partial least square regression (PLSR). The results showed $94 \%$ correct identification for blood and $14 \%$ false positives.

Edelman et al..$^{215}$ evaluated the feasibility of using NIR spectroscopy for blood stain identification and age estimation on dark backgrounds. Blood samples were deposited on white, black, red, green and blue cotton substrates. They also analyzed thirty substances that look like blood on white cotton. For the identification of bloodstains on colored cotton, a comparison to a library set of three bloodstains of different ages was made. The spectra of bloodstains of the first 77 days showed high correlation with the library data and were easily discriminated from the non-blood samples. For age estimation, the PLS regression was able to estimate successfully the bloodstains aged up to 1 month old, giving a root mean squared error of prediction of $8.9 \%$.

Li et $a l .{ }^{216}$ also used ATR-FTIR combined with PLS regression to determine the age of bloodstains on glass slides with blood that had been aged up to 107 days. The samples were stored under indoor and outdoor conditions, as similar as possible to a crime scene scenario. For the indoor condition, samples were exposed to direct sunlight during the day and no light at night. For the outdoor condition, samples were exposed to direct sunlight, heat, and humidity. Two PLS models (indoor and outdoor) with 7-85 days showed RMSEP values of 5.8 and 4.8 days, respectively. PLS-DA technique was also used to discriminate between fresh (age $\leq 1 \mathrm{~d}$ ) and older (age $>1$ d) bloodstains. The outdoor model showed better performance, discriminating fresh bloodstains, with a sensitivity value of 0.92 (compared to 0.25 of the indoor classification model), probably due to the more extreme conditions from the outdoors.

Zapata et al. ${ }^{217,218}$ published two studies with the aim of identifying and discriminating among different body fluid stains. In the first, ${ }^{217}$ they focused on discrimination among semen, vaginal fluid, and urine stains placed on different colored cotton fabrics using external reflection FTIR combined with SIMCA. The training set was composed of 18 stains from each fluid deposited on six cotton samples of different colors, 17 stains of semen, 17 stains of vaginal fluid, and 17 stains of urine (12 on white cotton from four different donors and five stains on each colored cotton). Several spectra were obtained for each stain. The external validation set consisted of six cotton samples of each color, three stains from each body fluid (from different donors than those used for the training set), 14 stains made by cosmetics and foodstuffs, and two stains of a mixture of semen and vaginal fluid. The SIMCA classification models showed correct classifications of all stains in the validation set. No false positives were obtained from the cosmetic or foodstuff substances. The stains containing a mixture of body fluids were classified as vaginal fluid. The authors highlighted that further research is needed to improve and refine the classification model, particularly with fluid mixtures where only the female's fluid was identified. In the second study, ${ }^{218}$ they used HSI-NIR to identify stains of semen, vaginal fluid, and urine on $100 \%$ cotton fabrics. The fluids were deposited on the substrates separately and a mixture of semen and vaginal fluid was also prepared. The PCA scores image made it possible to identify the location of each stain. CLS models were able to discriminate among the pixels related to semen and vaginal fluid on a mixture stain. It is important to remark, however, that CLS requires knowledge of all the compounds in the mixture, i.e., the fluids spectra and other compounds such as substrates and contaminants.

Takamura et al ${ }^{84,219}$ published two recent studies for discrimination of body fluids using ATR-FTIR and multivariate analysis. In the first, ${ }^{219}$ they raised important practical difficulties in the discrimination of body fluid using infrared spectroscopy: compositions of both body fluids and substrates are distributed heterogeneously on body fluid stains. In addition, body fluid compositions are diffused extensively into substrates thus making signals from body fluids weaker against strong substrate interference and more vulnerable to instrumental noise. As much forensic evidence cannot be anticipated, it is important that the discrimination method be applicable to unknown substrate types. To overcome these issues, the authors proposed a new strategy to recover blood signals in strong substrate interference situations using ATRFTIR spectroscopy and an innovative multivariate spectral processing. Postmortem blood (PB) and antemortem blood (AB) stains were used to show the efficacy of the method. The pre-processing approach uses data sets of pure blood samples of $\mathrm{PB}$ and $\mathrm{AB}$, obtained from dried stains on glass slides and pure substrate spectra. From spectra of PB and $\mathrm{AB}$ stains on interfering substrates (polyester, cotton, and denim), blood-originated signals were extracted using a weighted linear regression approach developed originally using PCs of both blood and substrate. The blood-originated signals were then classified by PLSDA using only the spectra of $\mathrm{PB}$ and $\mathrm{AB}$ on glass slides to build the models. GA was employed to evaluate the specific spectral regions for $\mathrm{PB}$ and $\mathrm{AB}$. As a result, the discrimination accuracy varied between 86 and $95 \%$, depending on the substrate.

In the second study, Takamura et al. ${ }^{84}$ discriminated among different body fluids, including peripheral blood, 
saliva, semen, urine and sweat. However, the substrate employed was not clearly specified. A PLS-DA model incorporating the LDA algorithm was successfully employed to identify the five fluids. However, as previously mentioned, both PLS-DA and LDA are hard modeling methods, which means that each sample will be assigned to one of the classes. This is a limitation in forensic contexts because it can give a variety of false-positives. To overcome this, the authors proposed a discrimination scheme, where a PLS-LDA model is built followed by Q-statistics tests for each class of body fluids, and any spectra not included in a class considered outliers and excluded. This strategy was successful with fourteen types of false-positive samples, including foodstuffs, cleaning products, among other outliers. Results showed high discriminatory performance for all classes, especially for blood samples. Additionally, the robustness of the models against aged body fluids was significantly improved using a discrimination scheme based on a dichotomous classification tree with hierarchical clustering. For this, they applied HCA to the PLS-DA scores of the first day and predicted by the first-day-five-class PLS-LDA-Q model. According to the structure of the dendrogram, a discriminant scheme was built, consisting of a dichotomous classification tree and Q-tests inserted at each end.

Silva et al. ${ }^{220}$ discriminated among human semen stains in fabrics of different colors and compositions made up from animal semen, breast milk and lubricants, using HSI-NIR and multivariate models. The authors were also hampered by the amount of influence of the substrate. They conducted an exhaustive data analysis to test the most suitable tool for the detection and discrimination of semen stains in diverse fabrics. PCA and MCR-ALS were used for initial identification and PLS-DA, sPLS-DA and SVM-DA were then used for classification. PCA was not successful due to the texture of fabrics that had high influence on the results. On the other hand, the MCR-ALS was able to identify the presence of stains on all fabrics and the recovered spectra showed high correlation with the pure spectrum of semen, independent of the fabric used as substrate. However, other compounds also showed high correlation with pure semen spectrum, which indicated that MCR-ALS could be used in a presumptive examination. Regarding the classification techniques, SVM-DA showed a better performance, in general, providing more specific models for all classes. However, sPLS-DA was the only technique that did not provide any false negatives for human semen. Given the fact that substrates may vary in real case scenarios and they may have a strong influence on the results, the authors advise the construction of models for each substrate.
Table 5 shows the references and the chemometric techniques employed for body fluids analysis.

As seen, confocal Raman using the $785 \mathrm{~nm}$ laser excitation was preferred for identification and discrimination of different body fluids, as well as age, race and gender determinations. Although the resolution curve fitting technique was preferred for establishing the Raman signature of the different fluids, the classification techniques PLS-DA, SVM-DA and SIMCA were used for discrimination issues. The calibration techniques PLSR and PCR were applied for age determinations. ATR-FTIR, HSI-NIR and portable NIR were preferred for identification and discrimination of different body fluids. The classification techniques PLS-DA, SVM-DA and SIMCA were used for IR data analysis and the calibration technique PLSR was preferred for age determinations.

\section{Conclusions}

After extensive literature research, the potential and advances made when combining the vibrational spectroscopy techniques with chemometric tools are undeniable. By demonstrating their ability, the techniques described above are expected to have a strong impact on forensic routines, since the majority of scientific police laboratories are equipped with either an infrared or a Raman spectrometer, and in many cases, both.

The chemometric techniques used will always vary according to the trace evidence and the protocol used to approach the problem in hands. Regarding unsupervised techniques, in general, PCA, as expected, is predominant in the majority of forensic investigations involving chemometrics. Although it is often erroneously referred as a classification technique, its ability to highlight differences and similarities are well established and explored not only for screening analysis, but also for decision-making. This is particularly important for document counterfeits, where there are many situations when discrimination of only two registers will elucidate the problem.

Regarding classification techniques, PLS-DA, LDA and SVM-DA are the most employed techniques for supervised classification, noting the special attention given to SVM-DA which is often employed for body fluids analysis. This might be due to the complexity of samples, the classification problem and, in some cases, the substrate influence. These issues lead to complex datasets that often need nonlinear boundaries for discrimination. In addition, it is extremely important to emphasize the need for research works that can combine forensic problems with different class-modeling techniques. Up to the moment, discriminant analysis dominates most forensic applications, but class- 
Table 5. Resume of techniques for body fluids

\begin{tabular}{|c|c|c|c|}
\hline Analytical technique & Chemometric technique & Sample & Reference \\
\hline Confocal Raman (785 nm) & curve fitting ${ }^{\mathrm{a}}$ & semen & 190 \\
\hline Confocal Raman (785 nm) & curve fitting $^{\mathrm{a}}$ & saliva & 191 \\
\hline Confocal Raman (785 nm) & curve fitting $^{\mathrm{a}}$ & blood & 192 \\
\hline Confocal Raman (785 nm) & curve fitting $^{\mathrm{a}}$ & sweat & 193 \\
\hline Confocal Raman (785 nm) & curve fitting ${ }^{\mathrm{a}}$ & vaginal fluid & 194 \\
\hline Confocal Raman (785 nm) & PCA, ${ }^{b}$ PLS-DA, ${ }^{c}$ SVM-DA ${ }^{c}$ & menstrual and peripheral blood from different donors & 195 \\
\hline Confocal Raman (785 nm) & PCA,${ }^{b}$ SIMCA,${ }^{c}$ LDA, ${ }^{c}$ PLS-DA ${ }^{c}$ & semen, blood and saliva from multiple donors & 196 \\
\hline Confocal Raman (785 nm) & $\begin{array}{l}\text { SVM-DA, }{ }^{c} \text { PLS-DA, }{ }^{c} \text { GA-SVM-DA, }{ }^{c} \\
\text { GA-PLS-DA, }{ }^{c}{ }^{\text {iPLS-DA }}{ }^{c}\end{array}$ & peripheral blood, saliva, semen, sweat and vaginal fluid & 197 \\
\hline Confocal Raman (785 nm) & SVM-DA, ${ }^{c} S M^{d}$ & mixtures of semen and blood & 198 \\
\hline Confocal Raman (785 nm) & $\mathrm{PCA}^{\mathrm{b}}$ & human, canine and feline blood & 199 \\
\hline Confocal Raman (785 nm) & PLS-DAc ${ }^{c}$ & human and animal blood from 11 species & 200 \\
\hline Confocal Raman (785 nm) & PLS-DA ${ }^{\mathrm{c}}$ & human and animal blood from 6 species & 201 \\
\hline Confocal Raman $(532,633,785 \mathrm{~nm})$ & PLS-DA ${ }^{c}$ & human and animal blood from 4 species & 202 \\
\hline Portable Raman (785 nm) & $\mathrm{PCA}^{\mathrm{b}}$ & aged human and animal blood from 11 species & 203 \\
\hline Confocal Raman (785 nm) & PCA, ${ }^{b} \mathrm{k}-\mathrm{NN},{ }^{\mathrm{c}} \mathrm{SVM}-\mathrm{DA},{ }^{\mathrm{c}} \mathrm{GA}-\mathrm{ANN}{ }^{\mathrm{c}}$ & male and female blood (gender determination) & 204 \\
\hline Confocal Raman (785 nm) & GA-SVM-DA, ${ }^{c}$ GA-ANN, ${ }^{c}$ SVM-DA ${ }^{c}$ & $\begin{array}{c}\text { Caucasian and African-American blood (race } \\
\text { determination) }\end{array}$ & 205 \\
\hline Confocal Raman (785 nm) & $\mathrm{SVM}^{-D A^{c}}$ & male and female saliva (gender determination) & 206 \\
\hline Confocal Raman (785 nm) & PLS-DA ${ }^{c}$ & blood contaminated with sand, dust and soil & 207 \\
\hline Confocal Raman (785 nm) & PLS-DAc & semen on 6 different substrates & 208 \\
\hline Confocal Raman (785 nm) & $\operatorname{PLSR}^{\mathrm{d}}$ & aged bloodstains on glass & 209 \\
\hline Confocal Raman (785 nm) & SVM-DA, ${ }^{\mathrm{c}}$ PLSR, ${ }^{\mathrm{d}} \mathrm{PCR}^{\mathrm{d}}$ & aged bloodstains on glass & 210 \\
\hline Confocal Raman (785 nm) & SVM-DA $^{c}$ & newborn, adolescent and adult blood & 211 \\
\hline ATR-FTIR & PLS-DAc & human, cat and dog blood & 212 \\
\hline Portable NIR & SIMCA, ${ }^{c}$ LDA,${ }^{c}$ PLS-DA ${ }^{c}$ & $\begin{array}{l}\text { human and animal blood and } 5 \text { red substances on } 4 \text { different } \\
\text { substrates }\end{array}$ & 213 \\
\hline Portable NIR & $\operatorname{PLSR}^{\mathrm{d}}$ & human blood and 8 red substances on 7 substrates & 214 \\
\hline NIR & $\mathrm{PLSR}^{\mathrm{d}}$ & $\begin{array}{l}\text { aged human blood and } 30 \text { red substances on cotton } \\
\text { substrates of different colors }\end{array}$ & 215 \\
\hline ATR-FTIR & PLSR, ${ }^{d}$ PLS-DA ${ }^{c}$ & aged (indoors and outdoors) human bloodstains on glass & 216 \\
\hline Reflection FTIR & SIMCA $^{\mathrm{c}}$ & semen, vaginal fluid and urine on colored fabrics & 217 \\
\hline HSI-NIR & $\mathrm{PCA}^{\mathrm{b}}{ }^{\mathrm{C}} \mathrm{CLS}^{\mathrm{d}}$ & semen, vaginal fluid and urine on cotton fabrics & 218 \\
\hline ATR-FTIR & PLS-DAc & $\begin{array}{l}\text { postmortem and antemortem blood on glass and different } \\
\text { fabrics }\end{array}$ & 219 \\
\hline ATR-FTIR & PLS-DA, ${ }^{c} \mathrm{HCA}^{\mathrm{b}}$ & $\begin{array}{l}\text { peripheral blood, saliva, semen, urine, sweat and } 14 \text { red } \\
\text { substances }\end{array}$ & 84 \\
\hline HSI-NIR & PCA, ${ }^{b}$ PLS-DA, ${ }^{c}$ SVM-DA, ${ }^{c}$ MCR-ALS ${ }^{\mathrm{a}}$ & semen on different fabrics & 220 \\
\hline
\end{tabular}

${ }^{a}$ Resolution; bexploratory; ${ }^{c}$ classification; ${ }^{d}$ calibration. PCA: principal component analysis; PLS-DA: partial least squares-discriminant analysis; SVM-DA: support vector machines-discriminant analysis; SIMCA: soft independent modeling of class analogy; LDA: linear discriminant analysis; GA: genetic algorithm; iPLS: interval partial least squares; k-NN: k-nearest neighbors; ANN: artificial neural networks; PLSR: partial least squares regression; PCR: principal component regression; ATR-FTIR: attenuated total reflectance-Fourier transform infrared spectroscopy; NIR: near infrared; HSI: hyperspectral images; CLS: classical least squares; HCA: hierarchical cluster analysis; MCR-ALS: multivariate curve resolution-alternating least squares.

modeling could be more adequate in certain cases, such as drugs and currency counterfeits, because the target class is highly controlled.
Different approaches for validation are being explored. Re-sampling techniques such as bootstrapping has becoming popular in forensic problems. This is particularly 
important because representativeness is hard to achieve in many cases and these approaches can be very useful. Another chemometric strategy that is also gaining acceptance is data fusion. Nevertheless, only low-level data fusion has been used with much success.

One major problem encountered in many applications is the substrate influence, especially in body fluids and GSR identification. The strategy to attenuate this problem depends, among other factors, on the analytical technique employed. Chemometric approaches have proven especially interesting, particularly pre-processing techniques, weighted least squares and MCR-ALS. The latter has an advantage due to its flexibility during model building and it can be applied to a variety of forensic problems when not all components present are well-known.

In a real scenario, the acceptance of chemometric models and the routine use of Raman spectroscopy is still a challenge. Due to the statistical background needed for model development, forensic experts still resist the employment and development of chemometric models in a daily basis. Naturally, it is expected that this scenario will change over the years, since the partnership between academic institutions and scientific police departments is increasing. In some particular cases, police officers have already employed chemometric models in their reports, which is an important step towards implementing chemometrics in real cases. Additionally, there are a number of other challenges that must be considered when implementing those models for a routine use. The maintenance of a particular model should be constantly improved, especially when complex samples are being analyzed.

Finally, it is important to understand that the future scenario for forensic analysis combined with vibrational spectroscopy and chemometrics is still gaining interest and being improved. The need for robust, fast and reliable models is still challenging, especially for in loco applications and well-established protocols of analysis.

\section{Acknowledgments}

The authors would like to acknowledge INCTAA (CNPq grants 573894/2008-6 and 465768/2014-8, and FAPESP grants 2008/57808-1 and 2014/50951-4), NUQAAPE-FACEPE (APQ-03461.06/14), Núcleo de Estudos em Química Forense (NEQUIFOR; CAPES AUXPE 3509/2014, Edital PROFORENSE 2014), CNPq (428891/2018-7), FACEPE (BFP-0800-1.06/17 and BCT-0305-1.06-17). The English text of this paper has been revised by Sidney Pratt, Canadian, MAT (The Johns Hopkins University), RSAdipTESL (Cambridge University).

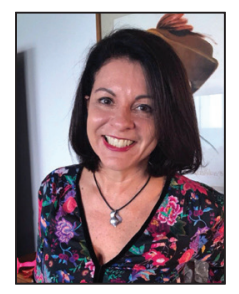

Maria Fernanda Pimentel obtained a Chemical Engineering degree in 1985, a master's degree in Chemistry in 1992 and a PhD in Chemistry in 1996 from the Federal University of Pernambuco (UFPE). She is a full professor at the Department of Chemical Engineering (UFPE) and coordinates the Development and Quality division of the Fuel Laboratory (LAC). Her research experience includes analytical chemistry/chemometrics with focus in multivariate analysis and calibration, design of experiments, infrared spectroscopy, forensic chemistry, quality control of fuels and process analytical technology.

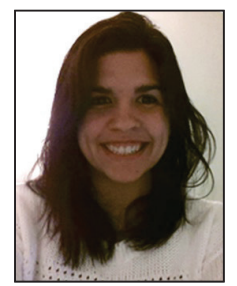

Carolina Santos Silva obtained a Chemistry degree in 2011, a master's degree in Chemistry in 2013, with an exchange period at the Polytechnic University of Valencia, and a PhD in Chemistry in 2017 from the Federal University of Pernambuco (UFPE), with an exchange period at the University of Copenhagen. Since then has been a postdoc at the Department of Chemical Engineering at UFPE. Her research experience includes analytical chemistry, infrared spectroscopy, multivariate analysis, hyperspectral image analysis and forensic chemistry.

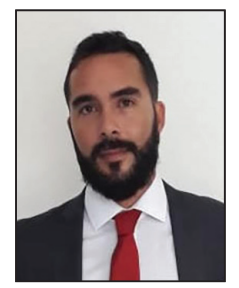

André Braz obtained a degree in Technological Chemistry from the University of Lisbon in 2005, a master's degree in Forensic Science from the University of Amsterdam in 2009 and a PhD in Criminalistics from the University of Alcalá in 2015. Since then has been a postdoc at the Department of Fundamental Chemistry of the Federal University of Pernambuco. His research experience includes forensic chemistry, Raman, infrared and UV-Vis spectroscopy, multivariate analysis and hyperspectral imaging.

\section{References}

1. Houck, M. M.; Forensic Chemistry, $1^{\text {st }}$ ed.; Academic Press: Oxford, UK, 2015.

2. Doty, K. C.; Muro, C. K.; Bueno, J.; Halámková, L.; Lednev, I. K.; J. Raman Spectrosc. 2016, 47, 39.

3. Doty, K. C.; Lednev, I. K.; TrAC, Trends Anal. Chem. 2018, 103, 215.

4. Payne, P.; Wallace, C.; Reedy, B.; Lennard, C.; Schuler, R.; Exline, D.; Roux, C.; Talanta 2005, 67, 334. 
5. Ewing, A. V.; Kazarian, S. G.; Analyst 2017, 142, 257.

6. Das, R. S.; Agrawal, Y. K.; Vib. Spectrosc. 2011, 57, 163.

7. de Araujo, W. R.; Cardoso, T. M. G.; da Rocha, R. G.; Santana, M. H. P.; Muñoz, R. A. A.; Richter, E. M.; Paixão, T. R. L. C.; Coltro, W. K. T.; Anal. Chim. Acta 2018, 1034, 1.

8. Burnett, A. D.; Cunningham, J. E.; Davies, A. G.; Dean, P.; Linfield, E. H. In Infrared and Raman Spectroscopy in Forensic Science; Chalmers, J. M.; Edwards, H. G. M.; Hargreaves, M. D., eds.; John Wiley \& Sons: West Sussex, UK, 2012, p. 295.

9. Muro, C. K.; Doty, K. C.; Bueno, J.; Halamkova, L.; Lednev, I. K.; Luber, C. K.; Doty, K. C.; Bueno, J.; Halámková, L.; Lednev, I. K.; Anal. Chem. 2015, 87, 306.

10. Martín-Alberca, C.; Ortega-Ojeda, F. E.; García-Ruiz, C.; Anal. Chim. Acta 2016, 928, 1.

11. Zadora, G.; Menżyk, A.; TrAC, Trends Anal. Chem. 2018, 105, 137.

12. Khandasammy, S. R.; Fikiet, M. A.; Mistek, E.; Ahmed, Y.; Halámková, L.; Bueno, J.; Lednev, I. K.; Forensic Chem. 2018, 8,111 .

13. Mazivila, S. J.; Olivieri, A. C.; TrAC, Trends Anal. Chem. 2018 , 108, 74.

14. Cailletaud, J.; Bleye, C.; Dumont, E.; Sacré, P. Y.; Netchacovitch, L.; Gut, Y.; Boiret, M.; Ginot, Y. M.; Hubert, P.; Ziemons, E.; J. Pharm. Biomed. Anal. 2018, 147, 458.

15. Yu, B.; Ge, M.; Li, P.; Xie, Q.; Yang, L.; Talanta 2019, 191, 1.

16. Kumar, R.; Sharma, V.; TrAC, Trends Anal. Chem. 2018, 105, 191.

17. Sharma, V.; Kumar, R.; TrAC, Trends Anal. Chem. 2018, 107, 181.

18. Bishop, C. M.; Pattern Recognition and Machine Learning; Springer: New York, USA, 2006.

19. Rinnan, Å.; Berg, F. V. D.; Engelsen, S. B.; TrAC, Trends Anal. Chem. 2009, 28, 1201.

20. Bocklitz, T.; Walter, A.; Hartmann, K.; Rösch, P.; Popp, J.; Anal. Chim. Acta 2011, 704, 47.

21. Lee, L. C.; Liong, C.; Jemain, A. A.; Chemom. Intell. Lab. Syst. 2017, 163, 64.

22. Wold, S.; Esbensen, K.; Geladi, P.; Chemom. Intell. Lab. Syst. 1987, 2, 37.

23. Bro, R.; Smilde, A.; Anal. Methods 2014, 2812.

24. Jain, A, K.; Murty, M. P.; Flynn, P. J.; ACM Comput. Surv. 1999, $31,264$.

25. Lee, I.; Yang, J. In Comprehensive Chemometrics; Brown, S. D.; Tauler, R.; Walczak, B., eds.; Elsevier: Amsterdam, Netherlands, 2009, p. 577.

26. Brereton, R.; Chemometrics: Data Analysis for the Laboratory and Chemical Plant; John Wiley \& Sons Ltd.: West Sussex, UK, 2003.

27. Miller, J. N.; Miller, J. C.; Statistics and Chemometrics for Analytical Chemistry, $6^{\text {th }}$ ed.; Pearson Education: Harlow, UK, 2010.
28. Friedman, J. H.; Tukey, J. W.; IEEE Trans. Comput. 1974, c-23, 881.

29. Hall, P.; Statistics 1989, 17, 589.

30. Huber, P.; Ann. Stat. 1985, 13, 435.

31. Jones, M. C.; Sibson, R.; J. R. Stat. Soc. Ser. A 1987, 150, 1.

32. Peña, D.; Prieto, F. J.; J. Am. Stat. Assoc. 2001, 96, 1433.

33. Peña, D.; Prieto, F.; Technometrics 2001, 43, 286.

34. Hou, S.; Wentzell, P. D.; Metabolomics 2014, 10, 589.

35. Hou, S.; Wentzell, P. D.; Anal. Chim. Acta 2011, 704, 1.

36. Hyvärinen, A.; Oja, E.; Neural Networks 2000, 13, 411.

37. Comon, P.; Signal Process. 1994, 36, 287.

38. Westad, F.; Kermit, M. In Comprehensive Chemometrics; Brown, S. D.; Tauler, R.; Walczak, B., eds.; Elsevier: Amsterdam, Netherlands, 2009, p. 227.

39. Wang, G.; Ding, Q.; Hou, Z.; TrAC, Trends Anal. Chem. 2008, 27,368 .

40. Næs, T.; Martens, H.; J. Chemom. 1988, 2, 155.

41. Kalivas, J. H. In Comprehensive Chemometrics; Brown, S. D.; Tauler, R.; Walczak, B., eds.; Elsevier: Amsterdam, Netherlands, 2009, p. 1.

42. Geladi, P.; Kowalski, B. R.; Anal. Chim. Acta 1986, 185, 1.

43. Wold, S.; Sjöström, M.; Eriksson, L.; Chemom. Intell. Lab. Syst. 2001, 58, 109.

44. Brereton, R. G.; Chemom. Intell. Lab. Syst. 2015, 149, 90.

45. Cao, K. A. L.; Boitard, S.; Besse, P.; BMC Bioinf. 2011, 12, 253.

46. Rinnan, Å.; Andersson, M.; Ridder, C.; Engelsen, S. B.; J. Chemom. 2014, 28, 439.

47. Norgaard, L.; Wagner, J.; Nielsen, J. P.; Munc, L.; Engelsen, S. B.; Appl. Spectrosc. 2000, 54, 413.

48. Wold, S.; Antti, H.; Lindgren, F.; Öhman, J.; Chemom. Intell. Lab. Syst. 1998, 44, 175.

49. Trygg, J.; Wold, S.; J. Chemom. 2002, 16, 119.

50. Hoffmann, I.; Filzmoser, P.; Serneels, S.; Varmuza, K.; J. Chemom. 2016, 30, 153.

51. Filzmoser, P.; Gschwandtner, M.; Todorov, V.; J. Chemom. 2012, $26,42$.

52. Rasmussen, M. A.; Bro, R.; Chemom. Intell. Lab. Syst. 2012, 119, 21.

53. Miller, C. E. In Process Analytical Technology; Bakeev, K. A., ed.; Blackwell Publishing: Oxford, UK, 2005, p. 226.

54. Roca-Pardiñas, J.; Cadarso-Suárez, C.; González-Manteiga, W. In Comprehensive Chemometrics; Brown, S. D.; Tauler, R.; Walczak, B., eds.; Elsevier: Amsterdam, Netherlands, 2009, p. 171.

55. Efron, B.; Tibshirani, R.; J. Am. Stat. Assoc. 1997, 92, 548.

56. Lee, L. C.; Liong, C. Y.; Jemain, A. A.; Microchem. J. 2018, 139, 125.

57. ASTM E1655-05: Standard Practices for Infrared Multivariate Quantitative Analysis; ASTM International, West Conshohocken, PA, 2005. 
58. Currie, L. A.; Anal. Chim. Acta 1999, 391, 127.

59. Olivieri, A. C.; Introduction to Multivariate Calibration: A Practical Approach; Springer: Cham, Switzerland, 2018.

60. Olivieri, A.; Escandar, G. M.; Practical Three-Way Calibration; Elsevier: Waltham, USA, 2014.

61. Faber, K.; Kowalski, B. R.; J. Chemom. 1997, 11, 181.

62. Widener, A.; Drahl, C.; Chem. Eng. News 2014, 92, 10.

63. Bevilacqua, M.; Bucci, R.; Magrì, A. D.; Magrì, A. L.; Marini, R. N. F. In Chemometrics in Food Chemistry; Marini, F., ed.; Elsevier: Oxford, UK, 2013, p. 498.

64. Ballabio, D.; Todeschini, R. In Infrared Spectroscopy for Food Quality Analysis and Control; Sun, D. W., ed.; Elsevier: Amsterdam, Netherlands, 2009, p. 83.

65. Oliveri, P.; Downey, G.; TrAC, Trends Anal. Chem. 2012, 35, 74.

66. Forina, M.; Oliveri, P.; Lanteri, S.; Casale, M.; Chemom. Intell. Lab. Syst. 2008, 93, 132.

67. Rodionova, O. Y.; Oliveri, P.; Pomerantsev, A. L.; Chemom. Intell. Lab. Syst. 2016, 159, 89.

68. Rodionova, O. Y.; Titova, A. V.; Pomerantsev, A. L.; TrAC, Trends Anal. Chem. 2016, 78, 17.

69. Oliveri, P.; Downey, G. In Comprehensive Analytical Chemistry, vol. 60; Guardia, M.; Gonzálvez, A., eds.; Elsevier: Amsterdam, Netherlands, 2013, p. 317.

70. Derde, M. P.; Massart, D. L.; Anal. Chim. Acta 1986, 184, 33.

71. Forina, M.; Armanino, C.; Leardi, R.; Drava, G.; J. Chemom. 1991, 5, 435.

72. Oliveri, P.; López, M. I.; Casolino, M. C.; Ruisánchez, I.; Callao, M. P.; Medini, L.; Lanteri, S.; Anal. Chim. Acta 2014, 851, 30.

73. Xu, L.; Goodarzi, M.; Shi, W.; Cai, C.; Jiang, J.; Chemom. Intell. Lab. Syst. 2014, 139, 58.

74. Wold, S.; Pattern Recognit. 1976, 8, 127.

75. Branden, K. V.; Hubert, M.; Chemom. Intell. Lab. Syst. 2005, 79,10 .

76. Louwerse, D. J.; Smilde, A. K.; Chem. Eng. Sci. 2000, 55, 1225.

77. Maesschalck, R.; Candolfi, A.; Massart, D. L.; Heuerding, S.; Chemom. Intell. Lab. Syst. 1999, 47, 65.

78. Kvalheim, O. M.; Øygard, K.; Grahl-Nielsen, O.; Anal. Chim. Acta 1983, 150, 145.

79. Daszykowski, M.; Kaczmarek, K.; Stanimirova, I.; Heyden, Y. V.; Walczak, B.; Chemom. Intell. Lab. Syst. 2007, 87, 121.

80. Pomerantsev, A. L.; J. Chemom. 2008, 22, 601.

81. Pomerantsev, A. L.; Rodionova, O. Y.; J. Chemom. 2014, 28, 429.

82. Zontov, Y. V.; Rodionova, O. Y.; Kucheryavskiy, S. V.; Pomerantsev, A. L.; Chemom. Intell. Lab. Syst. 2017, 167, 23.

83. Martins, A. R.; Talhavini, M.; Vieira, M. L.; Zacca, J. J.; Braga, J. W. B.; Food Chem. 2017, 229, 142.

84. Takamura, A.; Watanabe, K.; Tomoko, A.; Ozawa, T.; Sci. Rep. 2018, 8, 8459 .

85. Barker, M.; Rayens, W.; J. Chemom. 2003, 17, 166.
86. Fisher, R. A.; Ann. Eugen. 1936, 7, 179.

87. Lavine, B. K.; Rayens, W. S. In Comprehensive Chemometrics; Brown, S. D.; Tauler, R.; Walczak, B., eds.; Elsevier: Amsterdam, Netherlands, 2009, p. 517.

88. Araújo, M. C. U.; Saldanha, T. C. B.; Galvão, R. K. H.; Yoneyama, T.; Chame, H. C.; Visani, V.; Chemom. Intell. Lab. Syst. 2001, 57, 65.

89. Mehmood, T.; Liland, K. H.; Snipen, L.; Sæbø, S.; Chemom. Intell. Lab. Syst. 2012, 118, 62.

90. Jarvis, R. M.; Goodacre, R.; Bioinformatics 2005, 21, 860.

91. Smola, A. J.; Schölkopf, B.; Stat. Comput. 2004, 14, 199.

92. Brereton, R. G.; Lloyd, G. R.; Analyst 2010, 135, 230.

93. Suykens, J. In Comprehensive Chemometrics; Brown, S. D.; Tauler, R.; Walczak, B., eds.; Elsevier: Amsterdam, Netherlands, 2009, p. 437.

94. Ballabio, D.; Grisoni, F.; Todeschini, R.; Chemom. Intell. Lab. Syst. 2018, 174, 33.

95. López, M. I.; Callao, M. P.; Ruisánchez, I.; Anal. Chim. Acta 2015, 891, 62 .

96. Ellison, S. L. R.; Fearn, T.; TrAC, Trends Anal. Chem. 2005, 24,468

97. de Juan, A.; Jaumot, J.; Tauler, R.; Anal. Methods 2014, 6, 4964.

98. Maeder, M.; Anal. Chem. 1987, 59, 527.

99. Windig, W.; Stephenson, D. A.; Anal. Chem. 1992, 64, 2735.

100. Tauler, R.; Smilde, A.; Kowalski, B.; J. Chemom. 1995, 9, 31.

101. Jaumot, J.; Gargallo, R.; Juan, A.; Tauler, R.; Chemom. Intell. Lab. Syst. 2005, 76, 101.

102. Jaumot, J.; de Juan, A.; Tauler, R.; Chemom. Intell. Lab. Syst. 2015, 140, 1 .

103. Hugelier, S.; Piqueras, S.; Bedia, C.; de Juan, A.; Ruckebusch, C.; Anal. Chim. Acta 2017, 1000, 100.

104. Hugelier, S.; Firmani, P.; Devos, O.; Moreau, M.; Pierlot, C.; Marini, F.; Ruckebusch, C.; J. Spectral Imaging 2016, 5 , a7.

105. Hugelier, S.; Devos, O.; Ruckebusch, C.; J. Chemom. 2015, 29 , 557.

106. Juan, A.; Maeder, M.; Hancewicz, T.; Tauler, R.; Chemom. Intell. Lab. Syst. 2005, 77, 64.

107. Fikiet, M. A.; Khandasammy, S. R.; Mistek, E.; Ahmed, Y.; Halámková, L.; Bueno, J.; Lednev, I. K.; Spectrochim. Acta, Part A 2018, 197, 255.

108. Eliasson, C.; Macleod, N. A.; Matousek, P.; Anal. Chim. Acta 2008, 607, 50 .

109. Olds, W. J.; Sundarajoo, S.; Selby, M.; Cletus, B.; Fredericks, P. M.; Izake, E. L.; Appl. Spectrosc. 2012, 66, 530.

110. Petterson, I. E. I.; María, L.; Anal. Chem. 2011, 83, 8517.

111. Coates, J. P. In Process Analytical Technology: Spectroscopic Tools and Implementation Strategies for the Chemical and Pharmaceutical Industries; Bakeev, K. A., ed.; Blackwell Publishing: Oxford, UK, 2010, p. 91.

112. Pasquini, C.; Anal. Chim. Acta 2018, 1026, 8.

113. Pasquini, C.; J. Braz. Chem. Soc. 2003, 14, 198. 
114. Griffiths, P. R. In Infrared and Raman Spectroscopic Imaging; Salzer, R.; Siesler, H. W., eds.; Wiley-VCH: Weinheim, Germany, 2009, p. 3.

115. Prats-Montalbán, J. M.; de Juan, A.; Ferrer, A.; Chemom. Intell. Lab. Syst. 2011, 107, 1.

116. Malkoff, D. B.; Oliver, W. R. In Spectral Imaging: Instrumentation, Applications, and Analysis, vol. 3920; Bearman, G. H.; Cabib, D.; Levenson, R, M., eds.; SPIE: Bellingham, MA, USA, 2000, p. 108.

117. Amigo, J. M.; Babamoradi, H.; Elcoroaristizabal, S.; Anal. Chim. Acta 2015, 896, 34.

118. Grahn, H. F.; Geladi, P.; Techniques and Applications of Hyperspectral Image Analysis, $1^{\text {st }}$ ed.; Grahn, H. F.; Geladi, P., eds.; John Wiley \& Sons: West Sussex, UK, 2007.

119. Salzer, R.; Siesler, H.; Infrared and Raman Spectroscopic Imaging; Salzer, R.; Siesler, H., eds.; Wiley-VCH, Weinheim, Germany, 2009.

120. Calcerrada, M.; García-Ruiz, C.; Anal. Chim. Acta 2015, 853, 143.

121. Hoehse, M.; Paul, A.; Gornushkin, I.; Panne, U.; Anal. Bioanal. Chem. 2012, 402, 1443.

122. Almeida, M. R.; Correa, D. N.; Rocha, W. F. C.; Scafi, F. J. O.; Poppi, R. J.; Microchem. J. 2013, 109, 170.

123. Teixeira, C. A.; Poppi, R. J.; Microchem. J. 2019, 144, 411.

124. Braz, A.; López-López, M.; García-Ruiz, C.; Forensic Sci. Int. 2014, 245, 38.

125. Braz, A.; López-López, M.; García-Ruiz, C.; Forensic Sci. Int. 2015, 249, 92.

126. Borba, F. S. L.; Jawhari, T.; Honorato, S.; Juan, A.; Analyst 2017, 142, 1106.

127. Buzzini, P.; Polston, C.; Schackmuth, M.; J. Raman Spectrosc. 2018, 49, 1791 .

128. Silva, C. S.; Borba, F. S. L.; Pimentel, M. F.; Pontes, M. J. C.; Honorato, R. S.; Pasquini, C.; Microchem. J. 2013, 109, 122.

129. Silva, C. S.; Pimentel, M. F.; Honorato, R. S.; Pasquini, C.; Prats-Montalbán, J. M.; Ferrer, A.; Analyst 2014, 139, 5176.

130. Kher, A.; Mulholland, M.; Green, E.; Reedy, B.; Vib. Spectrosc. 2006, 40, 270.

131. Pereira, J. F. Q.; Silva, C. S.; Braz, A.; Pimentel, M. F.; Honorato, R. S.; Pasquini, C.; Wentzell, P. D.; Microchem. J. 2017, 130, 412.

132. Sharma, V.; Kumar, R.; Vib. Spectrosc. 2017, 92, 96.

133. Lee, L. C.; Liong, C. Y.; Jemain, A. A.; Chemom. Intell. Lab. Syst. 2018, 182, 90.

134. Asri, M. N. M.; Desa, W. N. S. M.; Ismail, D.; J. Forensic Sci. 2018, 63, 285.

135. Materazzi, S.; Risoluti, R.; Pinci, S.; Romolo, F. S.; Talanta 2017, 174, 673.

136. Kumar, R.; Sharma, V.; Spectrochim. Acta, Part A 2017, 175, 67.

137. Silva, C. S.; Pimentel, M. F.; Amigo, J. M.; García-Ruiz, C.; Ortega-Ojeda, F.; Anal. Chim. Acta 2018, 1031, 28.
138. Risoluti, R.; Materazzi, S.; Tau, F.; Russo, A.; Romolo, F. S.; Analyst 2018, 143, 4394.

139. Oliveira, V. S.; Saldanha, R.; Honorato, F. A.; Fernandes, C.; Forensic Sci. Int. 2018, 286, 121.

140. López-López, M.; Delgado, J. J.; García-Ruiz, C.; Anal. Chem. 2012, 84, 3581.

141. Maitre, M.; Kirkbride, K. P.; Horder, M.; Roux, C.; Beavis, A.; Forensic Sci. Int. 2017, 270, 1.

142. Bueno, J.; Sikirzhytski, V.; Lednev, I. K.; Anal. Chem. 2012 , 84, 4334.

143. Bueno, J.; Lednev, I. K.; Anal. Methods 2013, 5, 6292.

144. Bueno, J.; Lednev, I. K.; Anal. Bioanal. Chem. 2014, 406, 4595.

145. Bueno, J.; Sikirzhytski, V.; Lednev, I. K.; Anal. Chem. 2013, 85,7287

146. López-López, M.; de la Ossa, M. A. F.; Garcia-Ruiz, C.; Appl. Spectrosc. 2015, 69, 889.

147. Ortega-Ojeda, F. E.; Torre-Roldán, M.; García-Ruiz, C.; Talanta 2017, 167, 227.

148. Weber, I. T.; de Melo, A. J. G.; Lucena, M. A. D. M.; Rodrigues, M. O.; Alves Junior, S.; Anal. Chem. 2011, 83, 4720.

149. Lucena, M. A.; de Sá, G. F.; Rodrigues, M. O.; Alves, S.; Talhavini, M.; Weber, I. T.; Anal. Methods 2013, 5, 705.

150. Carvalho, M. A.; Talhavini, M.; Pimentel, M. F.; Amigo, J. M.; Pasquini, C.; Junior, S. A.; Weber, I. T.; Anal. Methods 2018, 10,4711 .

151. Huri, M. A. M.; Ahmad, U. K.; Ibrahim, R.; Omar, M.; Malays. J. Anal. Sci. 2017, 21, 267.

152. López-López, M.; García-Ruiz, C.; TrAC, Trends Anal. Chem. 2014, 54, 36.

153. Stewart, S. P.; Bell, S. E. J.; McAuley, D.; Baird, I.; Speers, S. J.; Kee, G.; Forensic Sci. Int. 2012, 216, e5.

154. López-López, M.; Ferrando, J. L.; García-Ruiz, C.; Anal. Chim. Acta 2012, 717, 92.

155. Hwang, J.; Park, A.; Chung, J. H.; Choi, N.; Park, J.-Q.; Cho, S. G.; Baek, S.-J.; Choo, J.; J. Mol. Struct. 2013, 1039, 130.

156. Gorte, B.; Stein, A.; IEEE Trans. Geosci. Remote Sens. 1998, 36, 803.

157. Almeida, M. R.; Correa, D. N.; Zacca, J. J.; Logrado, L. P. L.; Poppi, R. J.; Anal. Chim. Acta 2015, 860, 15.

158. Almeida, M. R.; Logrado, L. P. L.; Zacca, J. J.; Correa, D. N.; Poppi, R. J.; Talanta 2017, 174, 628.

159. Banas, K.; Banas, A.; Moser, H. O.; Bahou, M.; Li, W.; Yang, P.; Cholewa, M.; Lim, S. K.; Anal. Chem. 2010, 82, 3038.

160. Chen, T.; Schultz, Z. D.; Levin, I. W.; Analyst 2009, 134, 1902.

161. de la Ossa, M. Á. F.; Amigo, J. M.; García-Ruiz, C.; Forensic Sci. Int. 2014, 242, 228.

162. de la Ossa, M. Á. F.; García-Ruiz, C.; Amigo, J. M.; Talanta 2014, 130, 315.

163. Martín-Alberca, C.; Zapata, F.; Carrascosa, H.; Ortega-Ojeda, F. E.; García-Ruiz, C.; Talanta 2016, 149, 257. 
164. Risoluti, R.; Gregori, A.; Schiavone, S.; Materazzi, S.; Anal. Chem. 2018, 90, 4288.

165. Han, Z.; Liu, H.; Wang, B.; Weng, S.; Yang, L.; Liu, J.; Anal. Chem. 2015, 87, 4821.

166. Dong, R.; Weng, S.; Yang, L.; Liu, J.; Anal. Chem. 2015, 87, 2937.

167. Mabbott, S.; Eckmann, A.; Casiraghi, C.; Goodacre, R.; Analyst 2013, 138, 118.

168. Andreou, C.; Hoonejani, M. R.; Barmi, M. R.; Moskovits, M.; Meinhart, C. D.; ACS Nano 2013, 7, 7157.

169. D’Elia, V.; Rubio-Retama, J.; Ortega-Ojeda, F. E.; García-Ruiz, C.; Montalvo, G.; Colloids Surf., A 2018, 557, 43.

170. Dies, H.; Raveendran, J.; Escobedo, C.; Docoslis, A.; Sens. Actuators, B 2018, 257, 382.

171. Massarini, E.; Wästerby, P.; Landström, L.; Lejon, C.; Beck, O.; Andersson, P. O.; Sens. Actuators, B 2015, 207, 437.

172. Sondermann, N.; Kovar, K. A.; Forensic Sci. Int. 1999, 102, 133.

173. Sondermann, N.; Kovar, K. A.; Forensic Sci. Int. 1999, 106, 147.

174. Goh, C. Y.; van Bronswijk, W.; Priddis, C.; Appl. Spectrosc. 2008, 62, 640 .

175. Moros, J.; Galipienso, N.; Vilches, R.; Garrigues, S.; Guardia, M.; Anal. Chem. 2008, 80, 7257.

176. Pérez-Alfonso, C.; Galipienso, N.; Garrigues, S.; de la Guardia, M.; Forensic Sci. Int. 2014, 237, 70.

177. Grobério, T. S.; Zacca, J. J.; Botelho, É. D.; Talhavini, M.; Braga, J. W. B.; Forensic Sci. Int. 2015, 257, 297.

178. Kahmann, A.; Anzanello, M. J.; Fogliatto, F. S.; Marcelo, M. C. A.; Ferrão, M. F.; Ortiz, R. S.; Mariotti, K. C.; J. Pharm. Biomed. Anal. 2018, 152, 120.

179. Correia, R. M.; Domingos, E.; Tosato, F.; dos Santos, N. A.; Leite, J. D. A.; da Silva, M.; Marcelo, M. C. A.; Ortiz, R. S.; Filgueiras, P. R.; Romão, W.; Anal. Methods 2018, 10, 593.

180. Grobério, T. S.; Zacca, J. J.; Talhavini, M.; Braga, J. W. B.; J. Braz. Chem. Soc. 2014, 25, 1696.

181. Pérez-Alfonso, C.; Galipienso, N.; Garrigues, S.; Guardia, M.; Microchem. J. 2018, 143, 110.

182. Rodrigues, N. V. S.; Cardoso, E. M.; Andrade, M. V. O.; Donnici, C. L.; Sena, M. M.; J. Braz. Chem. Soc. 2013, 24, 507.

183. Marcelo, M. C. A.; Mariotti, K. C.; Ferrão, M. F.; Ortiz, R. S.; Forensic Sci. Int. 2015, 246, 65.

184. Liu, C.; Han, Y.; Min, S.; Jia, W.; Meng, X.; Liu, P.; Forensic Sci. Int. 2018, 290, 162.

185. Deconinck, E.; Van Campenhout, R.; Aouadi, C.; Canfyn, M.; Bothy, J. L.; Gremeaux, L.; Blanckaert, P.; Courselle, P.; Talanta 2019, 195, 142.

186. Risoluti, R.; Materazzi, S.; Gregori, A.; Ripani, L.; Talanta 2016, 153, 407.

187. Pereira, L. S. A.; Lisboa, F. L. C.; Neto, J. C.; Valladão, F.; Sena, M.; Microchem. J. 2017, 133, 96.
188. Pereira, L. S. A.; Lisboa, F. L. C.; Coelho Neto, J.; Valladão, F. N.; Sena, M. M.; Forensic Sci. Int. 2018, 288, 227.

189. Gondim, C. D. S.; Augusto, O.; Coelho, M.; Alvarenga, R. L.; Junqueira, R. G.; Vitorino, S.; Souza, C.; Anal. Chim. Acta 2014, 830, 11.

190. Virkler, K.; Lednev, I. K.; Forensic Sci. Int. 2009, 188, 1.

191. Virkler, K.; Lednev, I. K.; Analyst 2010, 135, 512.

192. Virkler, K.; Lednev, I. K.; Anal. Bioanal. Chem. 2010, 396, 525.

193. Sikirzhytski, V.; Sikirzhytskaya, A.; Lednev, I. K.; Anal. Chim. Acta 2012, 718, 78.

194. Sikirzhytskaya, A.; Sikirzhytski, V.; Lednev, I. K.; Forensic Sci. Int. 2012, 216, 44.

195. Sikirzhytskaya, A.; Sikirzhytski, V.; Lednev, I. K.; J. Biophotonics 2014, 7, 59.

196. Sikirzhytski, V.; Virkler, K.; Lednev, I. K.; Sensors 2010, 10, 2869.

197. Muro, C. K.; Doty, K. C.; Fernandes, L. D. S.; Lednev, I. K.; Forensic Chem. 2016, 1, 31.

198. Sikirzhytski, V.; Sikirzhytskaya, A.; Lednev, I. K.; Forensic Sci. Int. 2012, 222, 259.

199. Virkler, K.; Lednev, I. K.; Anal. Chem. 2009, 81, 7773.

200. Mclaughlin, G.; Doty, K. C.; Lednev, I. K.; Forensic Sci. Int. 2014, 238, 91.

201. Doty, K. C.; Lednev, I. K.; Forensic Sci. Int. 2018, 282, 204.

202. Bai, P.; Wang, J.; Yin, H.; Tian, Y.; Yao, W.; Gao, J.; Anal. Lett. 2016, 50, 379.

203. Fujihara, J.; Fujita, Y.; Yamamoto, T.; Nishimoto, N.; KimuraKataoka, K.; Kurata, S.; Takinami, Y.; Yasuda, T.; Takeshita, H.; Int. J. Leg. Med. 2017, 131, 319.

204. Sikirzhytskaya, A.; Sikirzhytski, V.; Lednev, I. K.; Anal. Chem. 2017, 89, 1486.

205. Mistek, E.; Halámková, L.; Doty, K. C.; Muro, C. K.; Lednev, I. K.; Anal. Chem. 2016, 88, 7453.

206. Muro, C. K.; Fernandes, L. D. S.; Lednev, I. K.; Anal. Chem. 2016, 88, 12489.

207. Sikirzhytskaya, A.; Sikirzhytski, V.; Mclaughlin, G.; Lednev, I. K.; J. Forensic Sci. 2013, 58, 1141.

208. Mclaughlin, G.; Lednev, I. K.; J. Forensic Sci. 2015, 60, 595. 209. Doty, K. C.; Muro, C. K.; Lednev, I. K.; Forensic Chem. 2017, 5 , 1.

210. Doty, K. C.; Mclaughlin, G.; Lednev, I. K.; Anal. Bioanal. Chem. 2016, 408, 3993.

211. Doty, K. C.; Lednev, I. K.; ACS Cent. Sci. 2018, 4, 862.

212. Mistek, E.; Lednev, I. K.; Anal. Bioanal. Chem. 2015, 407, 7435.

213. Pereira, J. F. Q.; Silva, C. S.; Vieira, M. J. L.; Pimentel, M. F.; Braz, A.; Honorato, R. S.; Microchem. J. 2017, 133, 561.

214. Morillas, A. V.; Gooch, J.; Frascione, N.; Talanta 2018, 184, 1.

215. Edelman, G.; Manti, V.; Van Ruth, S. M.; Van Leeuwen, T.; Aalders, M.; Forensic Sci. Int. 2012, 220, 239.

216. Lin, H.; Zhang, Y.; Wang, Q.; Li, B.; Huang, P.; Wang, Z.; Sci. Rep. 2017, 7, 13254. 
217. Zapata, F.; Ossa, M. A. F.; García-Ruiz, C.; Appl. Spectrosc. 2016, 70, 654.

218. Zapata, F.; Ortega-Ojeda, F. E.; García-Ruiz, C.; Talanta 2017, 166, 292.

219. Takamura, A.; Watanabe, K.; Akutsu, T.; Ikegaya, H.; Ozawa, T.; Anal. Chem. 2017, 89, 9797.
220. Silva, C. S.; Pimentel, M. F.; Amigo, J. M.; Honorato, R. S.; Pasquini, C.; TrAC, Trends Anal. Chem. 2017, 95, 23.

Submitted: February 15, 2019

Published online: June 18, 2019 